\title{
DINÁMICA POBLACIONAL DE GENETS Y RAMETS DE MAMMILLARIA GAUMERI CACTÁCEA ENDÉMICA DE YUCATÁN
}

\author{
Merari Ferrer, Rafael Durán', Martha Méndez, Alfredo Dorantes y Gabriel Dzib. \\ Centro de Investigación Científica de Yucatán \\ ${ }^{1}$ Autor para la correspondencia: rduran@cicy.mx.
}

\begin{abstract}
Resumen: Se analizó la dinámica poblacional de genets y ramets de Mammillaria gaumeri, cactácea endémica de Yucatán con el objetivo de generar información demográfica que sustente el cambio de estatus de especie de "protección especial" a "especie en peligro de extinción" en la Norma Oficial Mexicana. Se utilizó el modelo matricial para analizar la dinámica poblacional de ocho poblaciones por un periodo de cuatro años (2004-2007). Los resultados indican que las poblaciones están conformadas principalmente por adultos, con una densidad en el 2007 de 300 a 3,737 ind/ha. Se obtuvo un escaso porcentaje de germinación en campo y establecimiento de plántulas. La tasa de mortalidad fue mayor en las primeras categorías de tamaño, y cuando se registró alta mortalidad de adultos se debió a eventos de perturbación (saqueo ilegal, incendios). La tasa de crecimiento poblacional ( ) fluctuó de 0.6255 a 1.2629. Los valores de elasticidad indican que la permanencia de los adultos tuvo la mayor contribución a la tasa de crecimiento poblacional, por lo que la protección de estos individuos, así como la conservación del hábitat son medidas prioritarias para la permanencia de esta especie.
\end{abstract}

Palabras clave: análisis de elasticidad, demografía, Mammillaria, modelos matriciales.

\begin{abstract}
We analyzed the population dynamics of genets and ramets of Mammillaria gaumeri, an endemic cactus of Yucatan in order to generate demographic information that supports the change in status of species of "special protection" to "endangered species" in the Norma Oficial Mexicana. A matrix model was used to analyze the population dynamics of eight populations for a period of four years (2004-2007). The results indicate that the populations are comprised mainly of adults, with a density in 2007 from 300 to $3,737 \mathrm{ind} / \mathrm{ha}$. We obtained a low percentage of field germination and seedling establishment as reported for other globular cacti. The mortality rate was higher in the first categories of size, and when there was high mortality of adults due to disturbance events (illegal looting, fires). The population growth rate ( ) ranged from 0.6255 to 1.2629 . Elasticity values indicated that the stasis of adults had the greatest contribution to population growth rate, so that protection of these individuals, and its habitat conservation are priority actions for the survival of this species.
\end{abstract}

Keys words: demography, elasticity, Mammillaria, Matrix models.

$E^{n}$ las poblaciones de plantas clonales el reclutamiento de nuevos individuos puede ser por vía sexual y/o por propagación vegetativa. El reclutamiento vía sexual se presenta repetidamente en algunas poblaciones, mientras que en otras, es raro o esporádico, por lo que la dinámica poblacional de ciertas plantas clonales está dominada por el nacimiento y la muerte de los ramets producidos vegetativamente (Jordan y Nobel, 1979; Eriksson, 1989, 1993). Esto ocurre especialmente en ecosistemas áridos, donde la dinámica poblacional de las plantas varía considerablemente debido a las temperaturas extremas, a los impredecibles eventos de lluvia (Nobel, 1988; Valiente-Banuet y Ezcurra, 1991) y a la presión por herbivoría (Turner et al., 1969; McAuliffe, 1984; Cody,
1993). En estos ambientes y debido a estas condiciones, el establecimiento de plántulas de especies suculentas (como las cactáceas) es ocasional, y se restringe a pulsos durante los raros eventos de lluvia, aún cuando constantemente se produzcan semillas viables (Jordan y Nobel, 1979; Pierson y Turner, 1998; Mandujano et al., 1998). Por el contrario, la propagación clonal resulta ser un mecanismo exitoso que contribuye a la regeneración poblacional (Parker y Hamrick, 1992; Mandujano et al., 2001), y en ciertos casos, cuando el reclutamiento de plántulas es bajo o ausente, puede ser suficiente para asegurar la permanencia y el crecimiento de la población local (Abrahamson, 1980; Cook, 1985; Eriksson, 1993; Silvertown et al., 1993). 
El esfuerzo de propagación vegetativa, es uno de los resultados de la naturaleza modular de las plantas (Harper, 1977). En algunas especies estos módulos tienen la capacidad de generar su propio sistema radical y ser potencialmente independientes dando lugar a lo que se conoce como ramets, los cuales poseen la misma información genética que la planta madre (Harper, 1977; Abrahamson, 1980; Antonovics y Ellstrand, 1984; Harada et al., 1997). Además, la propagación vegetativa ha demostrado ser una estrategia muy exitosa en la naturaleza (Cook, 1983), ya que permite la persistencia exitosa de los genets y la colonización de nuevos ambientes a través de los ramets, sin pasar por las fases críticas de germinación y establecimiento, y confiere la capacidad de acaparar eficientemente el espacio y repartir el riesgo de muerte entre los ramets del mismo genet (Cook, 1983; Mendoza-Ochoa, 1994; García-Franco y Rico-Gray, 1995; Mandujano et al., 1998). Por otro lado, la reproducción sexual origina nuevas recombinaciones genéticas y la colonización de nuevos ambientes a través de la dispersión de las semillas a grandes distancias (Cook, 1985).

En la literatura existe mucha variación en la terminología sobre las plantas clonales, no obstante Harper y White (1974) señalan que el comportamiento poblacional de las especies de plantas que presentan propagación vegetativa, puede ser analizado a dos niveles: 1) a nivel de genets, donde la generación de nuevos ramets es considerada como una forma de crecimiento del genet (Harper, 1977, de Kroon et al., 2000), у 2) a nivel de ramets, donde la generación de clones se considera un proceso de generación de nuevos individuos, por lo que varios autores lo denominan un proceso de propagación o reproducción vegetativa (Mandujano et al., 1998, Mandujano-Sánchez, 2007).

Los estudios demográficos en plantas clonales se han enfocado en el estudio de la dinámica de los ramets, ya que el destino de los genets está directamente relacionado con el destino de los ramets que lo conforman (Silvertown y Lovett-Doust, 1993). Sin embargo, dichos estudios deben tomarse con cuidado ya que las tasas de natalidad y mortalidad de los ramets sólo afectan el tamaño y por tanto la adecuación del genet, mientras que las tasas de mortalidad y natalidad de los genets influyen directamente en el tamaño efectivo de las poblaciones teniendo importantes repercusiones ecológicas y evolutivas (Mendoza-Ochoa, 1994). También debe considerarse el efecto fisiológico de la integración entre las partes (ramets) (Schmid y Bazzaz, 1987), ya que existe una gran gama, tanto del grado de integración fisiológica entre los ramets, como del tiempo durante el cual permanecen unidos (Cook, 1983; Hartnett y Bazzaz, 1983; Mendoza-Ochoa, 1994; Klimeš et al., 1997). La integración de los ramets (interdependencia física y/o fisiológica) puede ser benéfica en ambientes donde los recursos son escasos (Grime, 1982), sin embargo, también puede afectar el crecimiento, la reproducción y la supervivencia de dichas plantas (Hartnett y Bazzaz, 1983; Mendoza-Ochoa, 1994).
Las cactáceas representan un interesante campo de estudio dado que al vivir en ambientes con condiciones extremas, han desarrollado una gran capacidad reproductiva, la cual consiste en un esfuerzo sexual destinado a producir semillas para la conquista permanente de nuevos hábitats, así como un esfuerzo asexual para la producción de hijuelos que aseguran su permanencia a largo plazo en los sitios colonizados (Mandujano et al., 1996, 2001). Sin embargo, el conocimiento sobre la estructura y la importancia relativa del reclutamiento clonal vs. sexual en el mantenimiento y crecimiento de las poblaciones de cactáceas clonales es todavía limitado debido a que las teorías ecológicas y evolutivas se han desarrollado para entender organismos unitarios (Harper, 1977).

Mammillaria gaumeri es una cactácea rara y endémica de la Península de Yucatán cuyo estudio poblacional es relevante porque es una de las especies de la región con mayor grado de vulnerabilidad, debido a su restringida distribución y especificidad de hábitat. Además, las actividades humanas y la consecuente perturbación de su hábitat han llevado a condiciones críticas a sus escasas poblaciones. Esta especie está catalogada actualmente en la Norma Oficial Mexicana (SEMARNAT, 2010) como especie "en peligro de extinción" y como especie "vulnerable" en El Libro Rojo de la Unión Internacional para la Conservación de la Naturaleza (IUCN, 1985).

Dado que son pocos los estudios poblacionales que han cuantificado la contribución relativa de la reproducción sexual y clonal en el crecimiento y mantenimiento de las poblaciones que presentan ambas formas de propagación (McFadden, 1991; Mendoza-Ochoa, 1994; Mandujano et al., 2001; Mondragón et al., 2004; Clark-Tapia et al., 2005), este estudio aborda la dinámica poblacional de genets y ramets de Mammillaria gaumeri utilizando para ello modelos matriciales.

\section{Materiales y Métodos}

Sitios de estudio. Este estudio se realizó en la costa norte de la Península de Yucatán, localizada al sureste de la República Mexicana. El clima de la costa es semiárido, con una temperatura media anual de $26{ }^{\circ} \mathrm{C}$, una máxima de $45^{\circ} \mathrm{C}$ y mínima de $10{ }^{\circ} \mathrm{C}$ (Thien et al., 1982). La precipitación anual es menor a los $700 \mathrm{~mm}$ y ocurre principalmente durante la época de lluvias (junio-octubre), con algunas lluvias aisladas en la época de "nortes" (noviembre-febrero), y una marcada estación seca que se presenta de marzo a mayo (Orellana et al., 1999). El estudio se desarrolló en ocho localidades, las cuales comprenden todo el rango de distribución de la especie en la costa norte peninsular y los dos tipos de vegetación donde crece: el matorral de duna costera y la selva baja caducifolia con cactáceas candelabriformes.

El matorral de duna costera presenta una mezcla de comunidades vegetales desde herbáceas, arbustivas y arbóreas (Durán y Olmsted,1999). La vegetación tiene una altura de 3 a $5 \mathrm{~m}$, y las especies más comunes son: Agave angus- 
tifolia, Bonellia macrocarpa, Bravaisia berlandieriana, Caesalpinia vesicaria, Cakile lanceolata, Canavalia rosea, Coccoloba uvifera, Coccothrinax readii, Metopium brownei, Opuntia dillenii, Pithecellobium keyense, Selenicereus donkelaarii, Sesuvium portulacastrum, Sideroxylon americanum y Thrinax radiata (Torres et al., 2010).

En la selva baja caducifolia con cactáceas candelabriformes (Miranda y Hernández-X., 1963) la vegetación alcanza alturas de 8 a $12 \mathrm{~m}$, y la mayoría de los árboles pierden sus hojas durante la época de secas. Algunas especies comunes son: Acacia gaumeri, A. pringlei, Apoplanesia paniculata, Bursera simaruba, Caesalpinia gaumeri, Diphysa carthagenensis, Guaiacum sanctum, Leucaena leucocephala, Lysiloma latisiliquum y Piscidia piscipula. Entre las especies de la familia Cactaceae encontramos a Acanthocereus tetragonus, Mammillaria gaumeri, Nopalea gaumeri, $N$. inaperta, Opuntia stricta, Pilosocereus gaumeri, Pterocereus gaumeri y Selenicereus donkelaarii (Durán et al., 1998). Cabe destacar que en este tipo de vegetación se presentan numerosas especies endémicas de la península de Yucatán (Espadas-Manrique et al., 2003).

Especie de estudio. Mammillaria gaumeri (Britton \& Rose) Orcutt, también conocida como Neomammillaria gaumeri (Britton \& Rose) o Mammillaria heyderi subsp. gaumeri (Britton \& Rose) D.R. Hunt (Anderson, 2001; Guzmán et al., 2003), es una cactácea globosa (Figura 1) que se distribuye únicamente en la costa norte de la Península de Yucatán. Habita exclusivamente en el matorral de duna costera y la selva baja caducifolia (Durán et al., 1998; López-Jiménez, 2001), siendo en la duna costera donde sus poblaciones suelen ser más abundantes.

Mammillaria gaumeri presenta de 8 a 13 series de tubérculos con forma espiral, posee de 12 a 14 espinas radiales ascendentes de 5 a $14 \mathrm{~mm}$ de longitud, rectas, blancas, con la punta de color café, y una espina central que puede medir hasta $10 \mathrm{~mm}$ de longitud, recta de color castaño, con tinte púrpura y punta negra. Sus flores son blancas, rosadas o amarillas, en forma de campana, de 12 a $14 \mathrm{~mm}$ de longitud (Bravo-Hollis y Sánchez-Mejorada, 1991). La floración ocurre de diciembre a mayo, con picos en la producción de flores en marzo y abril. El período de fructificación es de junio a diciembre, aunque en ocasiones se observan frutos maduros los primeros meses del año. La máxima producción de frutos ocurre en septiembre en sincronía con la temporada de lluvias. Se reproduce sexualmente, a través de semillas, y presenta un crecimiento reproductivo o propagación vegetativa por reiteración programada (Mandujano-Sánchez, 2007). En esta especie se distingue claramente la planta madre y sus ramets, la mayoría de las veces los ramets se ubican alrededor de la planta madre formando una suerte de corona, en tanto que en otras surgen de la base, cerca de la raíz de la planta. En esta especie el nivel de integración determina que los ramets se mantengan unidos a la planta madre prácticamente toda su vida, sin embargo ante la eventualidad de sufrir depredación, algún accidente, incendios o una enfermedad que provoque la muerte de la madre pueden subsistir como individuos independientes, disminuyendo el riesgo de muerte del genet en su conjunto. En todas las localidades de $M$. gaumeri es factible encontrar individuos que desarrollan propagación vegetativa y aquellos que se mantienen sin ella como genets solitarios.
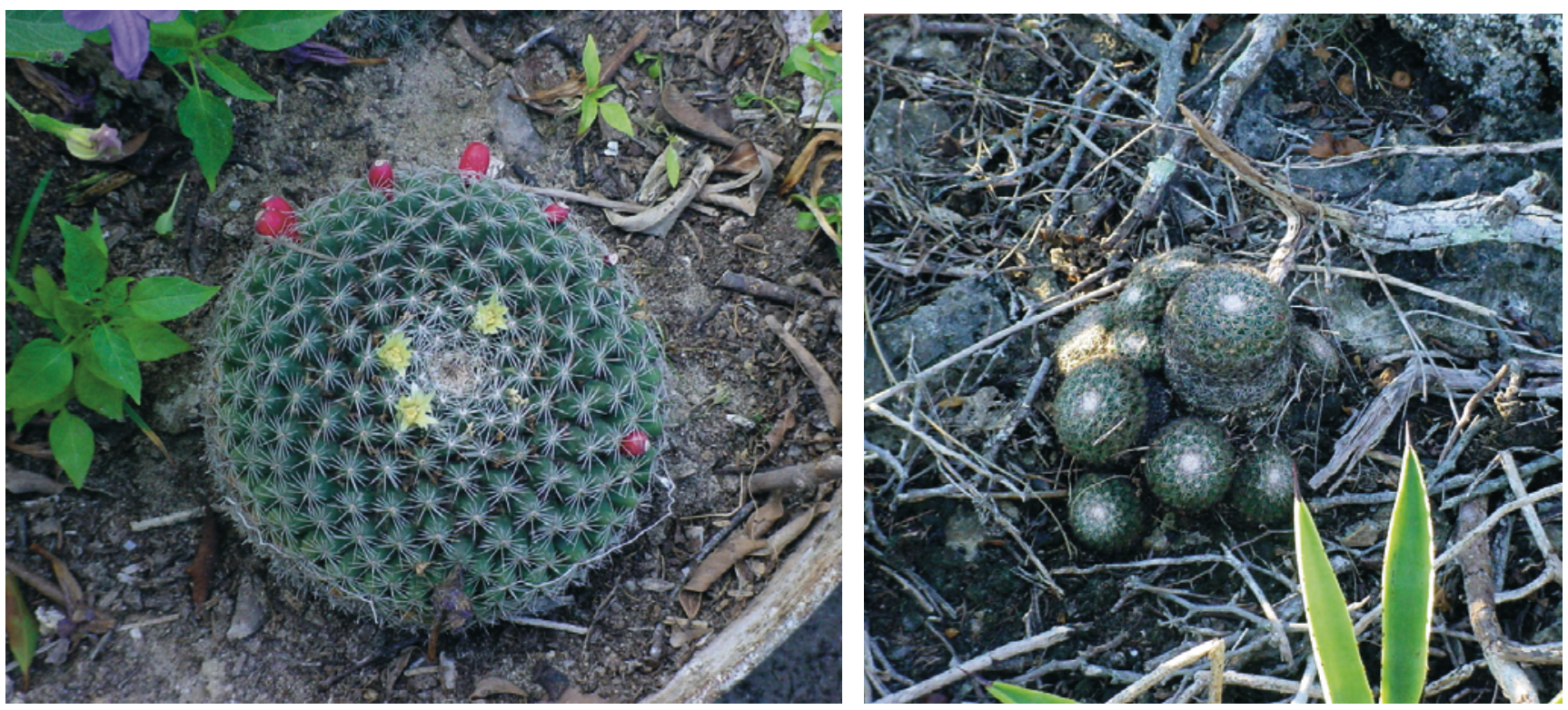

Figura 1. Individuos de Mammillaria gaumeri, en la imagen de la izquierda se observa un genet solitario y en la imagen de la derecha una planta madre con sus ramets. 


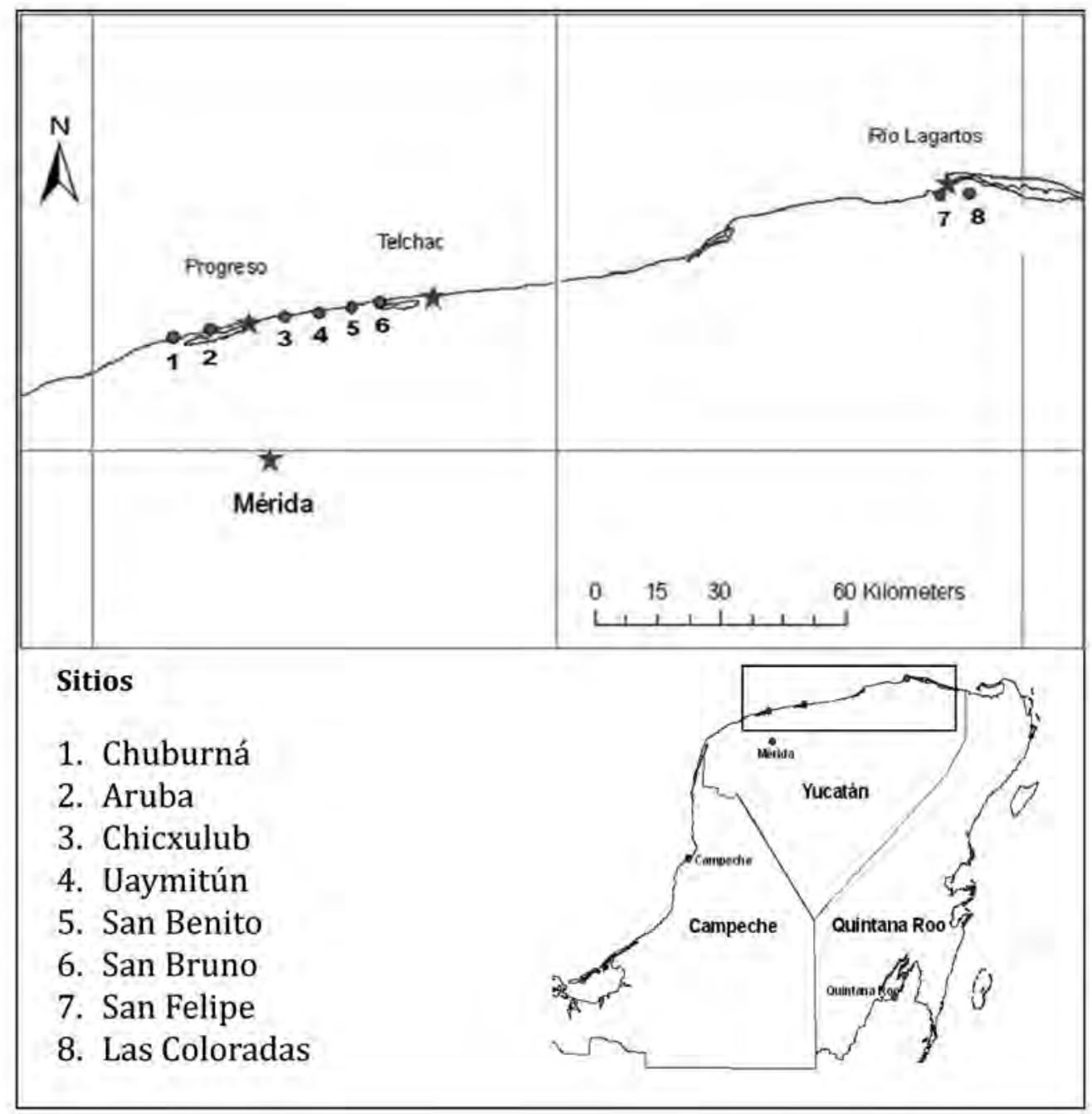

Figura 2. Ubicación de los sitios de muestreo de Mammillaria gaumeri. Las primeras seis poblaciones corresponden al matorral de duna costera y las dos últimas a la selva baja caducifolia.

Modelo demográfico. Los parámetros demográficos de Mammillaria gaumeri fueron estimados a través del modelo matricial poblacional (Lefkovitch, 1965; Caswell, 2001), ya que permite evaluar la importancia de la reproducción sexual vs. la propagación vegetativa en el mantenimiento de las poblaciones (Mandujano et al., 1998; Mondragón et al., 2004; Clark-Tapia et al., 2005). Para M. gaumeri se usaron matrices de tipo Lefkovitch $\mathrm{m} \times \mathrm{m}$, donde $\mathrm{m}(=10)$ es el número de filas y columnas consideradas. El modelo está definido por

$$
\mathbf{n}_{\mathrm{t}+1}=\mathbf{A} \cdot \mathbf{n}_{\mathbf{t}^{\prime}}
$$

donde A representa una matriz cuadrada y n es la columna vector que representa el número de individuos en cada categoría del tiempo $t$ al tiempo $t+1$. La matriz A está compuesta por $a_{\mathrm{ij}}$ entradas, las cuales representan la probabilidad de transición de una categoría de tamaño a otra en un período de tiempo (Caswell, 2001); de esta forma, en la matriz A los valores de la primera fila representan la contribución de cada estadio a la categoría de plántulas (fecundidad), los elementos de la diagonal principal representan la probabilidad de permanencia (estasis) o la proporción de individuos de la categoría i que permanecen en la misma categoría después de un intervalo de tiempo, los valores de la sub-diagonal son las probabilidades de transición o la proporción de individuos de una categoría de tamaño i que crecen a la siguiente categoría, y los elementos supra-diagonales representan el encogimiento o retroceso a categorías anteriores. En este estudio se construyeron matrices de proyección para las ocho poblaciones y tres períodos anuales (2004-2005, 2005-2006, 2006-2007; Apéndice 1).

Un complemento importante de los estudios matriciales es el análisis de elasticidad, porque evalúa cómo cambia el valor de cuando se modifica el valor de alguna de las entradas de la matriz (de Kroon et al., 2000), y dado que la suma de las entradas de la matriz de elasticidad es uno es 


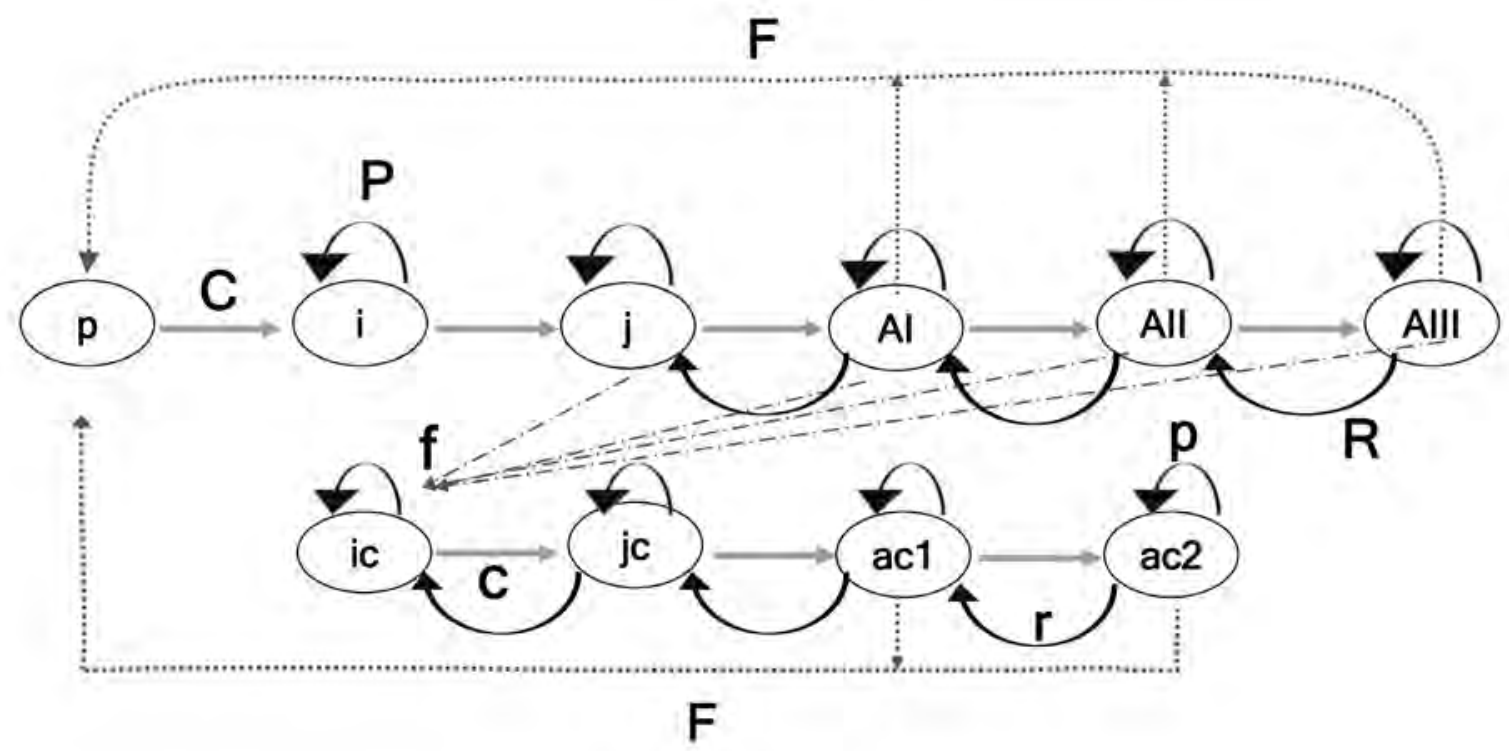

Figura 3. Ciclo de vida de Mammillaria gaumeri. Las letras en mayúscula corresponden a los procesos de individuos de origen vía semilla y las minúsculas a los procesos de origen vegetativo. Las flechas representan los posibles destinos que pueden tener los individuos dentro de cada estadio entre un año y otro: sobrevivir sin transitar al siguiente estadio (P) y (p), crecer lo suficiente para cambiar de estadio (C) y (c), retrogresión a categorías inferiores $(\mathrm{R})$ y (r) y reproducirse a través de la producción de plántulas (F) o de hijuelos (f).

posible sumar diferentes regiones de la matriz, las cuales representan los diferentes procesos demográficos (permanencia, crecimiento, reproducción), o las diferentes categorías de tamaño del ciclo de vida (Silvertown et al., 1993). Con este análisis, se determinó qué categorías de tamaño de Mammillaria gaumeri son las más vulnerables, y a qué proceso demográfico lambda ( ) es más sensible.

Estudio de campo. Se estudiaron seis poblaciones de Mammillaria gaumeri correspondientes al matorral de duna costera y dos a la selva baja con cactáceas candelabriformes (Figura 2). En cada sitio se establecieron 3 parcelas de muestreo permanente de $100 \mathrm{~m}^{2}$ cada uno $(10 \times 10 \mathrm{~m})$, donde se localizaron, marcaron y mapearon todos los individuos de M. gaumeri presentes. Las poblaciones fueron monitoreadas en el mes de noviembre por cuatro años consecutivos (20042007). En cada censo anual, se midió el diámetro y la altura de cada individuo presente (genets y ramets).

Categorías de clasificación. Los individuos se clasificaron por categorías de tamaño en función de su diámetro y algunas características fenológicas. Dado que se espera que las tasas de crecimiento, reproducción y supervivencia de los individuos provenientes de semillas sean diferentes de aquellos originados asexualmente, se procedió a establecer categorías para los individuos de diferente origen. Genets: plántulas $\leq 1 \mathrm{~cm}$; infantiles $=1.01-2.0 \mathrm{~cm}$; juveniles $=2.01$ $-4.0 \mathrm{~cm}$; adulto $\mathrm{I}=4.01-7.0 \mathrm{~cm}$; adulto $\mathrm{II}=7.01-10.0$ $\mathrm{cm}$; adulto $\mathrm{III} \geq 10.01 \mathrm{~cm}$. Ramets: infantil clonal $\leq 2.0 \mathrm{~cm}$; juvenil clonal $=2.01-4.0 \mathrm{~cm}$; adulto clonal $1=4.01-7.0$ $\mathrm{cm}$; adulto clonal $2=7.0-10.0 \mathrm{~cm}$. Se establecieron en total diez categorías de tamaño, seis para los individuos originados vía semillas y cuatro para los individuos originados por propagación vegetativa (Figura 3).

Germinación y establecimiento de plántulas. Los porcentajes de germinación bajo condiciones naturales se determinaron mediante experimentos de campo. Estos experimentos se montaron en el mes de septiembre por tres años consecutivos (2004-2006) en dos poblaciones, una de matorral de duna costera y una de selva baja caducifolia. Se introdujeron semillas de Mammillaria gaumeri bajo diferentes condiciones y/o tratamientos de germinación en campo: sitios sombreados/sitios expuestos al sol; semillas desprotegidas/ semillas protegidas a la depredación. La germinación de las semillas se evaluó diariamente durante 15 días, y después mensualmente por un año. Debido al bajo número de plántulas encontrado en gran parte de las poblaciones, se decidió evaluar durante un año la supervivencia de las plántulas obtenidas en los experimentos de germinación en campo para estimar la transición plántulas-infantiles.

Estimación de los parámetros demográficos. Para estimar la fecundidad (definida como el número de plántulas promedio que cada individuo adulto produce durante un año), se realizaron observaciones sobre la fenología reproductiva (producción de flores y frutos) y el porcentaje de germinación en campo de Mammillaria gaumeri. De manera que la fecundidad por categoría, con base en el número de plántulas per cápita, se calculó mediante la siguiente fórmula:

$$
\mathrm{F}_{\mathrm{ij}}=f \cdot \mathrm{s} \cdot g / n
$$

donde $f$ es el número total de frutos producido por los in- 
Merari Ferrer, Rafael Durán, Martha Méndez, Alfredo Dorantes y Gabriel Dzib.

Cuadro 1. Características demográficas de las poblaciones de Mammillaria gaumeri.

\begin{tabular}{|c|c|c|c|c|c|c|c|c|}
\hline Población & +Chuburná & +Aruba & +Chicxulub & +Uaymitún & +San Benito & +San Bruno & *San Felipe & *Las Coloradas \\
\hline $\begin{array}{l}\text { Densidad genets } \\
\text { (ind./ha) } 2007\end{array}$ & 300 & 1,500 & 2,133 & 1,137 & 3,737 & 467 & 1,033 & 533 \\
\hline Genets 2007 & 9 & 45 & 64 & 34 & 113 & 14 & 31 & 16 \\
\hline Ramets 2007 & 28 & 23 & 43 & 22 & 117 & 21 & 26 & 83 \\
\hline $\begin{array}{l}\% \text { genets con hijuelos } \\
2007\end{array}$ & 21.6 & 16.4 & 14.4 & 8.6 & 21.5 & 49 & 26 & 81.2 \\
\hline $\begin{array}{l}\% \text { genets adultos } \\
\text { reproductivos** }\end{array}$ & 41 & 41 & 61 & 74 & 68 & 73 & 31 & 89 \\
\hline $\begin{array}{l}\% \text { ramets adultos } \\
\text { reproductivos** }\end{array}$ & 29 & 13 & 0 & 27 & 33 & 13 & 14 & 75 \\
\hline
\end{tabular}

dividuos del estadio analizado, multiplicado por el número promedio de semillas producido por fruto $(s)$, y por la probabilidad de germinación en campo $(g)$, por último, el valor obtenido es dividido entre el número total de individuos dentro del estadio analizado (n). Para estimar la fecundidad se utilizó el porcentaje de germinación obtenido en los tratamientos excepto en aquellos protegidos de la depredación.

El número de flores y frutos producidos por individuo se determinó mediante la evaluación de todas las poblaciones durante un año (2005-2006) a partir de observaciones directas. Para estimar el número de semillas promedio por fruto, se colectaron 95 frutos fuera de las parcelas de estudio, procurando que todas las categorías adultas estuvieran representadas. La fecundidad por propagación vegetativa per cápita por estadio adulto, se estimó contabilizando el número total de hijuelos producidos al año por los individuos de cada categoría, luego se dividió esta cantidad entre el número total de individuos pertenecientes a dicha categoría.

Las tasas de permanencia se calcularon en función de la proporción de individuos de cada categoría que sobrevivieron pero que no alcanzaron el diámetro suficiente para cambiar de categoría durante ese año. Los valores de transición se estimaron de acuerdo a la proporción de individuos de cada categoría que, a lo largo de un año, alcanzó el diámetro suficiente para pasar a otra categoría o que decreció lo suficiente para entrar dentro del intervalo de diámetros de una categoría inferior. La probabilidad de transición plántulasinfantiles no pudo ser estimada de los experimentos de supervivencia en campo dado el escaso número de plántulas germinadas; en estos casos se asignó un valor arbitrario a la matriz (0.001) asumiendo que sólo una de 1,000 plántulas avanza a la siguiente categoría. Particularmente para la localidad de San Benito, existe un reporte sobre la germinación y el establecimiento de plántulas de Mammillaria gaumeri, por lo que se utilizó esta información, donde el porcentaje de germinación fue de $4.8 \%$ y la supervivencia de plántulas fue de 1.6\% (López-Haas, 2007).
Análisis de datos. La tasa de crecimiento de la población ( ), la estructura estable de edades $(w)$, el valor reproductivo $(v)$, y el análisis de elasticidad fueron calculados con el programa Gaussi. La estructura estable de edades se comparó con la distribución observada usando la prueba de G (Zar, 1984). Los límites de confianza de fueron estimados con el método analítico propuesto por Álvarez-Buylla y Slatkin (1991):

$$
V()={ }_{i, j} V\left(a_{i, j}\right) s\left(a_{i, j}\right)^{2}+{ }_{i, j}={ }_{k, j} \operatorname{Cov}\left(a_{i j}, a_{k j}\right) \cdot s\left(a_{i j}\right) \cdot s\left(a_{k j}\right)
$$

donde $V()$ es la varianza de lambda, $V\left(a_{\mathrm{ij}}\right)$ es la varianza asociada a dicha entrada, $s$ representa el valor de sensibilidad de la entrada y $\operatorname{Cov}\left(a_{\mathrm{ij}}, a_{\mathrm{kj}}\right)$ es la covarianza que existe entre cada par de entradas localizadas en una misma columna. El valor de las varianzas asociadas a las entradas de permanencia y transición tienen una distribución binomial por lo que su varianza $(V)$ es:

$$
V\left(a_{i j}\right)=a_{i j} \cdot\left(1-a_{i j}\right) / n
$$

donde $n$ es el número total de individuos dentro del estadio de la entrada analizada. La varianza asociada a las entradas de fecundidad se obtiene directamente de la variación observada en los datos de campo. De igual forma, el valor de la covarianza es estimada con la siguiente fórmula:

$$
\operatorname{Cov}\left(a_{i j}, a_{k j}\right)=\left(-\left(a_{i j}\right) \cdot\left(a_{k j}\right)\right) / n
$$

el error estándar ( ) se calculó como la raíz cuadrada de la varianza de . Los intervalos de confianza al $95 \%$ fueron calculados como \pm 2 .

\section{Resultados}

Abundancia y estructura poblacional. En el 2004 se marcaron un total de 866 individuos: 455 genets y 431 ramets; para el 2007 el número de genets disminuyó a 339 individuos y el de ramets a 364 . Tres poblaciones presentaron mayor número de ramets: Chuburná, San Bruno y Las Coloradas, en tanto que San Benito presenta prácticamente el mismo número de genets y ramets; el resto de las poblaciones están conformadas en su mayor parte por genets. Las Coloradas es la pobla- 

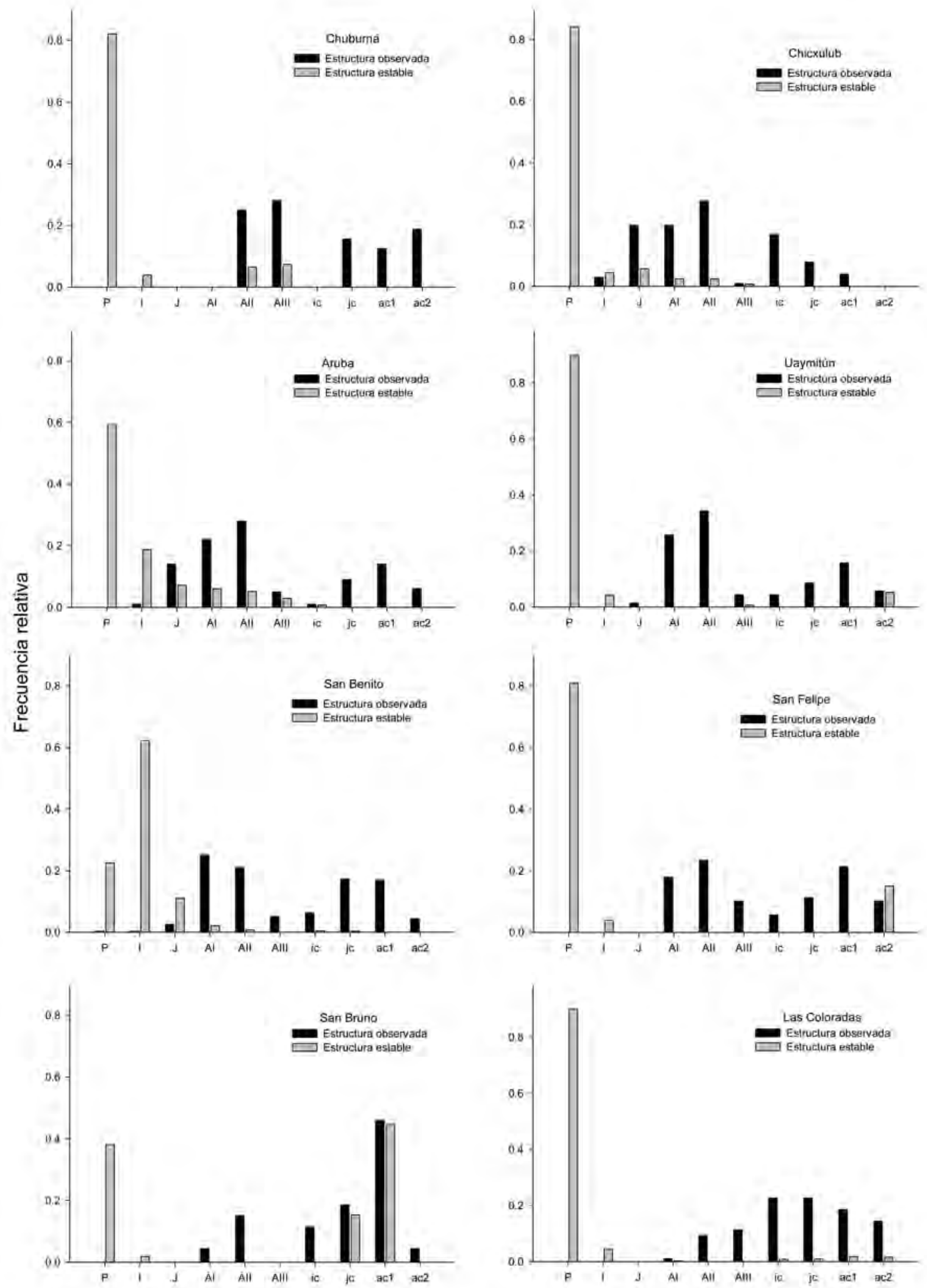

Estadios del ciclo de vida

Figura 4. Estructura poblacional observada y la estructura estable para cada población de Mammillaria gaumeri en el 2004. Las diferencias entre las frecuencias observadas y esperadas fueron significativas $(G=23.13, P<0.0001) . \mathrm{P}=$ plántula, $\mathrm{I}=$ infantil, $\mathrm{J}=$ juvenil, AI $=$ adulto I, AII = adulto II, AIII = adulto III; ic = infantil clonal, jc = juvenil clonal, ac1 = adulto clonal 1 , ac2 = adulto clonal 2. 


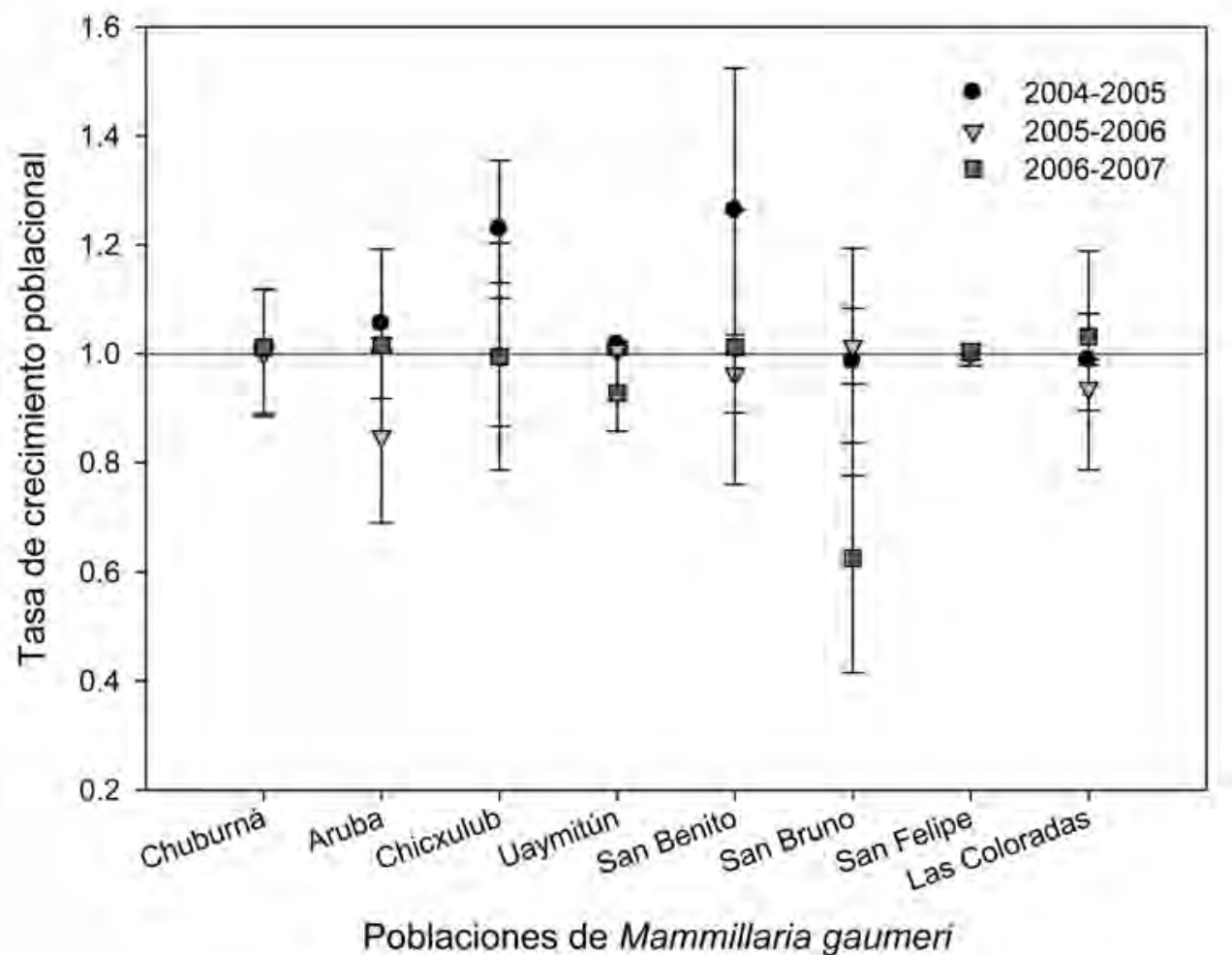

Figura 5. Tasa de crecimiento poblacional (con sus intervalos de confianza) de las ocho poblaciones estudiadas de Mammillaria gaumeri del 2004 al 2007.

ción que presentó el mayor porcentaje de genets con hijuelos $(83 \%)$, mientras que Uaymitún presenta el mayor porcentaje de genets solitarios (91.4\%) para el 2007 (Cuadro 1).

En las poblaciones de Mammillaria gaumeri las categorías de tamaño que comprenden a los adultos (de ambos orígenes) son las más abundantes (92\% de los individuos son adultos), y dentro de los adultos las categorías AI y AII abarcan la mayor proporción de individuos (Figura 4). Las primeras categorías de tamaño están prácticamente ausentes en las poblaciones de esta especie. Las categorías infantil y juvenil tienen una mayor representación vía clonal que vía semillas.

Germinación y establecimiento de plántulas. Los porcentajes de germinación encontrados en campo durante los tres años de evaluación fueron muy bajos en ambos tipos de vegetación, y las semillas sólo germinaron bajo condiciones sombreadas. En el 2004, para la duna costera, el porcentaje de germinación bajo condiciones sombreadas y expuestas a la depredación fue de $2.9 \%$ ( $\mathrm{N}=1,500$ semillas), ninguna plántula sobrevivió a lo largo del año siguiente. En el 2005 el porcentaje de germinación fue de $1.1 \%(\mathrm{~N}=525)$; al cabo de un año sólo una plántula sobrevivió. En el 2006 el porcentaje de germinación fue de $1.91 \%(\mathrm{~N}=525)$ y no hubo supervivencia de plántulas. Los bajos porcentajes encontrados no permitieron realizar un análisis estadístico de este experimento.

Fecundidad. Los individuos de Mammillaria gaumeri co- mienzan a reproducirse cuando alcanzan los $4 \mathrm{~cm}$ de diámetro. En la producción de frutos 2005-2006 los genets fueron más productivos que los ramets, de manera que los genets contribuyeron con el $81 \%$ de la producción total, mientras que los ramets contribuyen con el $19 \%$. La población Las Coloradas presentó el mayor número de individuos reproductivos, tanto de genets como ramets (Cuadro 1). La producción anual de frutos (2005-2006) fue de 3,227 frutos, con un rango de variación de 1,081 frutos en la población San Benito a 61 frutos en Chuburná. La producción promedio anual por individuo adulto reproductivo fue de diez frutos, siendo los adultos AII (de 7-10 cm de diámetro) los más productivos con 1, 497 frutos, lo que representa el $46 \%$ de la producción total.

El número de semillas encontradas en los frutos varió de 4 a 135 semillas, con un valor promedio para todas las poblaciones de 72 semillas por fruto. Las categorías que se reproducen vegetativamente son los adultos AI, AII y AIII, aunque se observaron algunos juveniles con hijuelos. El estadio AII es el más productivo y contribuye con el $61.7 \%$ de la producción de hijuelos, seguida por los adultos AI $(19.5 \%)$ y los AIII (15.6\%).

Mortalidad. Las primeras categorías de tamaño de Mammillaria gaumeri presentaron la mayor mortalidad. La tasa de mortalidad de las categorías juvenil (J) y adultos (AI, AII, AIII) fue menor, sin embargo, en algunos años, se registra- 

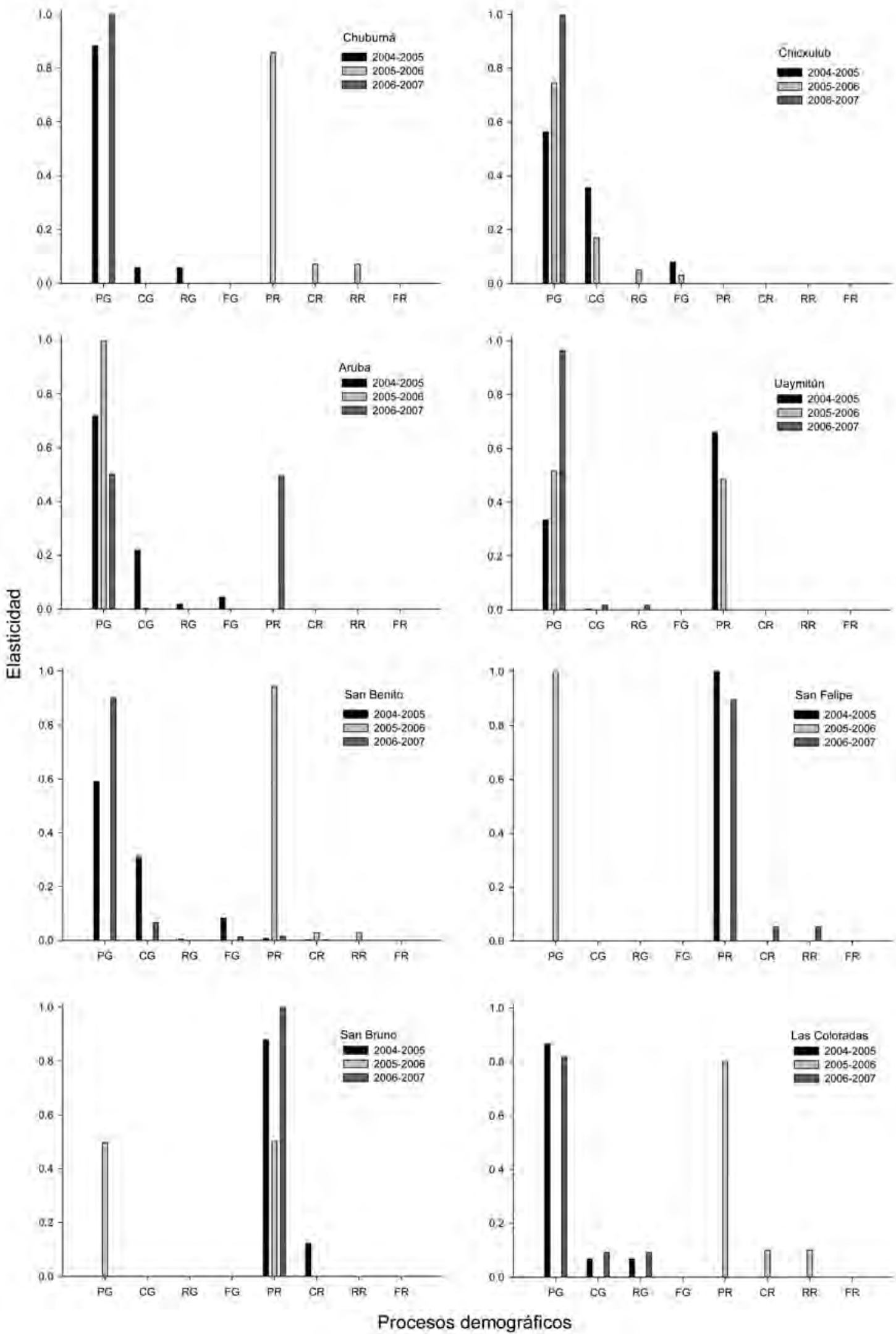

Figura 6. Valores de elasticidad de los procesos demográficos. $\mathrm{PG}=$ permanencia genets, $\mathrm{CG}=$ crecimiento genets, $\mathrm{RG}=$ retrogresión genets, $\mathrm{FG}=$ fecundidad genets, $\mathrm{PR}=$ permanencia ramets, $\mathrm{CR}=$ crecimiento ramets, $\mathrm{RR}=$ retrogresión ramets, $\mathrm{FR}=$ fecundidad ramets. 

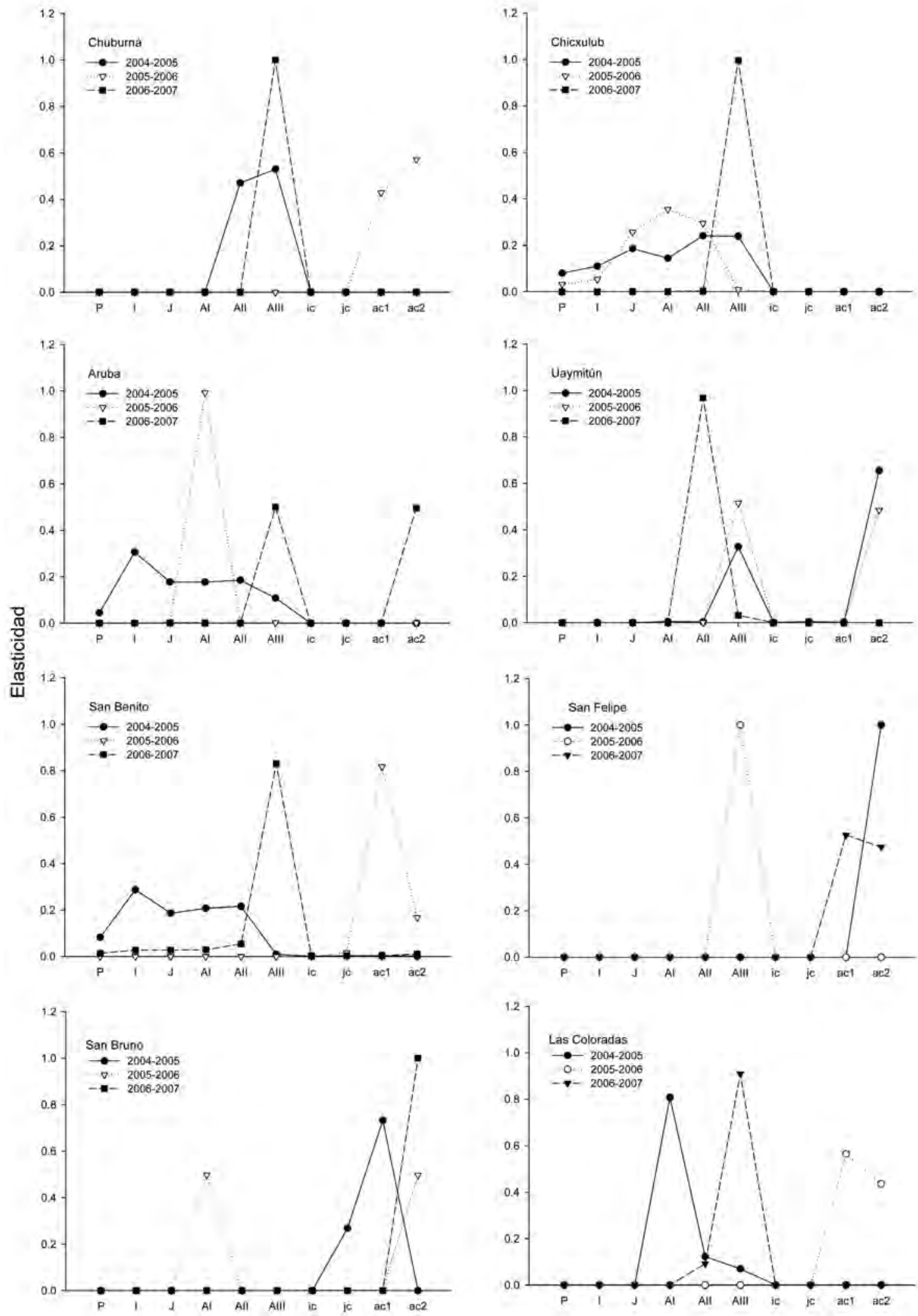

Procesos demográficos

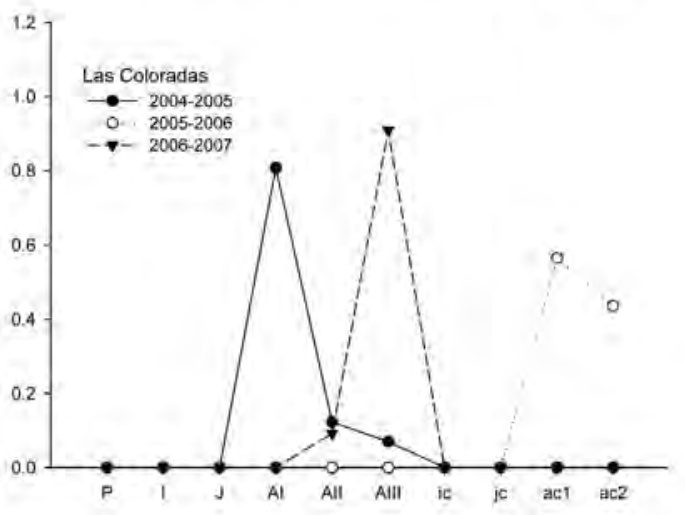

Estadios del ciclo de vida

Figura 7. Valores de elasticidad de las estadios del ciclo de vida de Mammillaria gaumeri. $\mathrm{P}=$ plántula, $\mathrm{I}=$ infantil, $\mathrm{J}=$ juvenil, $\mathrm{AI}=$ Adulto I, AII = Adulto III, AIII = Adulto III; ic = infantil clonal, jc = juvenil clonal, ac1 = adulto clonal 1, ac2 = adulto clonal 2. 
ron altas tasas de mortalidad de estas categorías asociadas a eventos de perturbación. Por ejemplo, se registró un alto porcentaje de mortalidad de adultos AII (38\%) y AIII (63\%) en Chuburná (2005) debido a la extracción de individuos de las parcelas experimentales; lo mismo ocurrió en Aruba ese mismo año. El año de mayor mortalidad fue el 2007, principalmente para San Bruno, donde murió el $73 \%$ de los individuos, como consecuencia de los incendios provocados en la localidad.

Tasa de crecimiento poblacional y estructura estable de tamaños. Las poblaciones de Mammillaria gaumeri presentaron tasas de crecimiento poblacional cercanas a la unidad, con valores de que van de 0.6255 a 1.2629 . Los intervalos de confianza (95\%) indican que la mayoría de los valores de lambda obtenidos no difieren significativamente de la unidad (Figura 5). Las excepciones ocurren en el 2004 en Chicxulub y San Benito, donde se registraron valores significativamente superiores a la unidad; y en San Bruno (2005-2006), Las Coloradas (2006-2007) y en Uaymitún (2006-2007) con tasas de crecimiento significativamente menores a uno, indicando el decremento del tamaño poblacional (Apéndice 1).

En todas las poblaciones y para todos los años, los valores de la estructura estable $(w)$ difiere significativamente de las estructuras iniciales observadas en campo $(G=23.13, P$ $<0.0001)$. La estructura estable $(w)$ predice un mayor número de plántulas e infantiles (categorías de menor tamaño), lo cual difiere con la estructura encontrada en campo, donde estas categorías están prácticamente ausentes. Los mayores valores reproductivos $(v)$ fueron para los individuos adultos de ambos orígenes, y de éstos, los genets presentaron un mayor valor reproductivo que los ramets (Apéndice 1).

Análisis de elasticidad. La elasticidad por proceso demográfico señala a la permanencia $(\mathrm{P} \approx 1)$ como el parámetro que tiene la mayor contribución sobre la tasa de crecimiento poblacional. Los valores de elasticidad considerando los diferentes procesos demográficos, en función del origen de los individuos, muestran que en la mayoría de las poblaciones y entre años de estudio, el valor de lambda depende de la permanencia de los individuos originados por semillas (genets), aunque, hay años donde el valor de lambda está determinado principalmente por la permanencia de los ramets, como ocurre en Chuburná, San Benito y Las Coloradas (2005-2006), San Bruno y San Felipe (Figura 6). Este análisis muestra que los cambios en los valores de permanencia, especialmente de los adultos AII y AIII y ac2, tiene el mayor impacto en la tasa de crecimiento poblacional (Figura 7). La suma de las elasticidades en función del origen de los individuos (genets y ramets) indica que el valor de lambda está determinado en su mayoría por el destino de los individuos originados vía semillas. No obstante hay poblaciones en las que difiere la contribución de genets y ramets entre años, sobre todo en aquellas poblaciones que presentan mayor nú- mero de ramets.

\section{Discusión}

La densidad más alta encontrada en este estudio (3,737 ind/ ha) fue baja comparada con la densidad encontrada para Mammillaria crucigera (10,000 individuos/ha) (Contreras y Valverde, 2002) y alta comparada con la densidad reportada para M. magnimamma (600 individuos/ha) (Valverde et al., 1999). Puede atribuirse que esta densidad es el resultado de diversos factores, entre los cuales destacan los fenómenos naturales, como los huracanes, y el efecto de diversas actividades humanas como la ganadería, los incendios, la construcción de casas y caminos, así como la extracción ilegal de los individuos de las poblaciones. La duna costera en Yucatán ha sido severamente perturbada en las últimas décadas, llegando a disminuir en más de la mitad de su extensión original, siendo la pérdida de hábitat y la deforestación para el desarrollo y construcción de casas de verano las causas principales. En la selva baja, la principal afectación se debe al desarrollo de la ganadería extensiva, lo cual ha originado una gran fragmentación del hábitat, por lo que actualmente el área está ocupada por un mosaico vegetal con parches en diferentes estados sucesionales. Una consecuencia de esta afectación ha sido la desaparición de algunas poblaciones de esta especie. López-Jiménez, en el 2001 reportó la existencia de 12 poblaciones de $M$. gaumeri en la costa de Yucatán, de las cuales sólo ocho sobreviven a la fecha y son objeto de este estudio.

La estructura poblacional de Mammillaria gaumeri difirió significativamente de la estructura estable. El patrón general observado indica que los individuos en las primeras categorías (plántulas, infantiles y juveniles) son menos frecuentes que aquellos de las categorías más avanzadas. La mayor proporción de individuos se concentró en la categoría adulta, lo que puede estar indicando que en años recientes ha habido un decremento en la tasa de reclutamiento en estas poblaciones, y/o que los ciclos de reclutamiento y de establecimiento para esta especie ocurre por pulsos (Steenbergh y Lowe, 1969; Jordan y Nobel, 1979; Franco y Nobel, 1989; Valiente-Banuet y Ezcurra, 1991).

Además, la estructura poblacional encontrada coincide con las estructuras encontradas en otras cactáceas globosas, como Mammillaria crucigera y M. magnimamma, donde la categoría de adultos es la más abundante y las plántulas son las menos representadas (Contreras y Valverde, 2002; Valverde et al., 2004). Otro aspecto importante a considerar para esta especie es la reproducción clonal o propagación vegetativa. En este aspecto, en la mayoría de las poblaciones la proporción de genets es mayor a la de ramets, a excepción de San Bruno y Las Coloradas, donde el número de ramets supera el de los genets.

En Mammillaria gaumeri la floración y fructificación ocurre durante la estación seca, y la maduración de los fru- 
Merari Ferrer, Rafael Durán, Martha Méndez, Alfredo Dorantes y Gabriel Dzib.

tos y la dispersión de semillas están sincronizadas con el inicio de la estación lluviosa; sin embargo, el porcentaje de germinación en campo resultó ser muy bajo comparado con los porcentajes reportados en laboratorio (98\%) (LópezHaas, 2007). La alta mortalidad de plántulas es un factor que afecta el reclutamiento de individuos y que puede estar limitando la distribución y abundancia de M. gaumeri. Después de un año sólo una plántula sobrevivió de 525 semillas colocadas en campo. Godínez-Álvarez et al. (2003) señalan que la alta radiación y los cambios drásticos de temperatura, contribuyen al bajo porcentaje de germinación y reclutamiento de plántulas en otras especies de cactáceas (Franco y Nobel, 1989; Valiente-Banuet et al., 1991; Godínez-Álvarez et al., 1999; Esparza-Olguín et al., 2002; Méndez et al., 2004). Por su parte Cervera et al. (2006) mencionan que $M$. gaumeri presenta requerimientos ambientales específicos de temperatura y humedad, así como la presencia de plantas nodriza para su germinación y establecimiento.

Las primeras categorías del ciclo de vida son las que presentaron las mayores tasas de mortalidad, y una vez que se supera la fase crítica de plántula-infantil, la tasa de mortalidad es relativamente constante para el resto de los categorías, a menos que ocurra un evento de perturbación. Este patrón de mortalidad también se ha reportado para cactáceas globosas como Mammillaria crucigera (Contreras y Valverde, 2002), M. magnimamma (Valverde et al., 2004), Coryphantha robbinsorum (Schmalzel et al., 1995), y para cactáceas columnares como Pterocereus gaumeri (Méndez et al., 2004) y Neobuxbaumia macrocephala (Esparza-Olguín et al., 2002), donde la mortalidad es mayor en las primeras categorías de tamaño.

Eriksson (1993) menciona que las plantas clonales usualmente exhiben baja fecundidad y reclutamiento de plántulas. En el caso de Mammillaria gaumeri, el reclutamiento de individuos vía hijuelos o ramets fue menor que vía semillas. Un aspecto a destacar es que la mayor contribución de frutos es por parte de los genets, no obstante, la fecundidad vía hijuelos también presenta una contribución importante en el mantenimiento de las poblaciones de esta especie. Sin embargo, el conocimiento sobre el papel de la reproducción sexual vs. la propagación clonal en el mantenimiento y crecimiento de poblaciones que crecen en ambientes áridos y semiáridos es todavía muy limitado. Clark-Tapia et al. (2005) estudiaron la importancia de la propagación vegetativa en la dinámica poblacional de Stenocereus eruca, una cactácea columnar del desierto de Sonora, encontrando que el reclutamiento clonal es más importante que el establecimiento de plántulas en la dinámica de esta especie, ya que cuando simulaban la remoción de la contribución sexual en las matrices de transición, no había un impacto en el valor de lambda, mientras que la ausencia de la propagación clonal fue suficiente para que el valor de lambda estuviera por debajo de la unidad. En el caso de M. gaumeri, la propagación clonal de esta especie no es tan determinante en la dinámica de las poblaciones, ya que los mayores valores de permanencia, crecimiento y fecundidad encontrados en las matrices de transición se deben a los genets. Sin embargo, los valores de elasticidad muestran que hay años, donde precisamente la permanencia de los ramets es determinante en la dinámica poblacional, sobre todo cuando ocurren eventos de perturbación como incendios o huracanes.

Las tasas de crecimiento de las poblaciones de Mammillaria gaumeri están cercanas a la unidad, por lo que se supone que las poblaciones de esta especie están en equilibrio. Estos valores concuerdan con lo reportado para otras cactáceas globosas como M. crucigera (Contreras y Valverde, 2002) y M. magnimmama (Valverde et al., 2004), y para cactáceas columnares como Carnegiea gigantea (Pierson y Turner, 1998), Neobuxbaumia tetetzo (Godínez-Álvarez et al., 1999), N. macrocephala (Esparza-Olguín et al., 2002), Pterocereus gaumeri (Méndez et al., 2004). El bajo valor de lambda obtenido en San Bruno (2007) que fue significativamente diferente de la unidad, puede ser explicado por la alta tasa de mortalidad encontrada en ese año, ya que la población fue quemada y se perdió el $73 \%$ de los individuos.

La permanencia de los individuos en la misma categoría fue el proceso demográfico con mayor contribución al valor de $(\mathrm{P} \approx 1)$, en particular la de los individuos adultos, lo cual es consistente con las tendencias reportadas para la familia Cactaceae y para otras especies de vida larga (Godínez-Álvarez et al., 1999; Mandujano et al., 2007). Si bien, en este estudio, la permanencia de los adultos originados vía semilla tuvo la mayor contribución al valor de , en algunos años la permanencia de los ramets fue determinante, lo cual indica la importancia de la propagación clonal para las poblaciones de esta especie sobre todo ante la ocurrencia de eventos catastróficos. Los resultados también indican la importancia de proteger a los adultos, especialmente en las poblaciones con valores bajos de . Esta protección está también relacionada con la producción de semillas, ya que los adultos más grandes producen más flores, frutos y semillas. Los esfuerzos de conservación también deben incluir la preservación de la estructura de la comunidad donde habita esta especie para mantener los microhábitats óptimos para la germinación y supervivencia de plántulas como sugiere Cervera et al. (2006).

De acuerdo a los resultados obtenidos, las poblaciones de Mammillaria gaumeri están cercanas al equilibrio demográfico y en condiciones naturales podrían permanecer en el tiempo. Sin embargo, el incremento de las actividades humanas ha colocado a esta especie en una situación de riesgo, debido a la fragmentación y pérdida del hábitat, lo que ha afectado no sólo el tamaño de las poblaciones sino que ha conducido a la extinción local a algunas de ellas. Las quemas, la fragmentación y el establecimiento de basureros clandestinos, no sólo afecta negativamente a los individuos de $M$. gaumeri, sino que también tiene impacto sobre las posibles nodrizas a través de la pérdida de la vegetación, 
afectando la germinación y el reclutamiento de nuevos individuos. La conservación de esta especie sólo será posible si se detienen los procesos de fragmentación, cambio de uso de suelo y la extracción de los individuos, que les afectan.

La mayoría de los estudios demográficos de cactáceas muestra que las poblaciones tienden a comportarse de forma estable (Godínez-Álvarez et al., 2003), e incluso son capaces de recuperarse después de eventos catastróficos como huracanes e incendios, tal como ocurre con Mammillaria gaumeri. Estas especies pueden mantenerse a lo largo del tiempo, a pesar de las estresantes condiciones en las que habitan. Sin embargo, es claro que numerosas especies de esta familia se encuentran en condiciones de riesgo (SEMARNAT, 2010), situación determinada por las diversas actividades antropogénicas que les afectan directa e indirectamente (Contreras y Valverde, 2002; Esparza-Olguín et al., 2002; Méndez et al., 2004; Valverde et al., 2004; Mandujano et al., 2007).

Por otra parte, se requiere tomar medidas para promover la conservación del germoplasma de esta especie, a través de la colecciones ex situ, la propagación de individuos en invernaderos, ya sea para su reintroducción a los hábitats naturales y/o para su venta comercial, lo que ayudaría a diminuir el saqueo de sus poblaciones. Estas medidas deben llevarse a cabo junto con programas de educación ambiental que permitan a los habitantes de la región costera apreciar a esta especie, su hábitat y hacer un uso sustentable de ésta.

Finalmente, los resultados de este trabajo sirvieron de base para proponer, mediante el Método de Evaluación de Riesgo de extinción de las especies silvestres de México (MER), el cambio de estatus de Mammillaria gaumeri de "protección especial" a "especie en peligro de extinción", en la Norma Oficial Mexicana que establece la protección ambiental de las especies nativas de México. Este cambio ya fue aprobado, tal como aparece en la NOM-059-SEMARNAT-2010, publicada en el Diario Oficial de la Federación el 30 de diciembre del 2010.

\section{Agradecimientos}

Este trabajo fue financiado por CONACYT-Apoyo a investigadores en proceso de consolidación: P52953. FerrerCervantes Merari Esther fue becada por CONACYT No. 166998. Agradecemos a los revisores sus comentarios para el enriquecimiento de este manuscrito.

\section{Literatura citada}

Abrahamson W.C. 1980. Demography and vegetative reproduction. En: Solbring O.T. Ed. Demography and Evolution in Plant Populations, pp. 89-106, University of California Press, Berkeley.

Álvarez-Buylla E.R. y Slatkin M. 1991. Finding confidence intervals of population growth rates. Trends in Ecology \& Evolution 6:221-224.
Anderson E.F. 2001. The Cactus Family. Timber Press, Portland.

Antonovics J. y Ellstrand N.C. 1984. Experimental studies of the evolutionary significance of sexual reproduction. I. A test of the frecuency-dependent selection hypothesis. Evolution 38:103-115.

Bravo-Hollis H. y Sánchez-Mejorada-R H. 1991. Las Cactáceas de México Vol. II. Universidad Nacional Autónoma de México, México, D.F.

Caswell H. 2001. Matrix Population Models. Sinauer Associates Inc., Sunderland.

Cervera J.C., Andrade J.L., Simá J.L., Graham E.A. 2006. Microhabitats, germination and establishment for Mammillaria gaumeri (Cactaceae), a rare species from Yucatan. International Journal of Plant Sciences 167:311-319.

Clark-Tapia R., Mandujano M.C., Valverde T., Mendoza A. y Molina-Freaner F. 2005. How important is clonal recruitment for population maintenance in rare plant species? The case of the narrow endemic cactus, Stenocereus eruca, in Baja California, Mexico. Biological Conservation 124:123-132.

Cody M.L. 1993. Do Cholla cacti (Opuntia spp., subgenus Cylindropuntia) use or need nurse in the Mojave desert? Journal of Arid Environments 24:139-154.

Contreras C. y Valverde T. 2002. Evaluation of the conservation status of a rare cactus (Mammillaria crucigera) through the analysis of its population dynamics. Journal of Arid Environments 51:89-102.

Cook R.E. 1983. Clonal plant populations: A knowledge of clonal structure can affect the interpretation of data in a broad range of ecological and evolutionary studies. American Scientist 71:244-253.

Cook R.E. 1985. Growth and development in clonal plant populations. En: Jackson J.B.C., Buss L.W. y Cook R.E. Eds. Populations Biology and Evolution of Clonal Organisms, pp.259-296, Yale University Press, New Haven.

de Kroon H.,van Groenendael J. y Ehrlén J. 2000. Elasticities: a review of methods and model limitations. Ecology 81:607-618.

Durán R. y Olmsted I. 1999. Vegetación de la Península de Yucatán. En: García de Fuentes A. y Córdoba-Ordoñez J. Eds. Atlas de Procesos Territoriales de Yucatán, pp.187-194, Universidad Autónoma de Yucatán, Mérida.

Durán R., Trejo-Torres J.C. e Ibarra-Manríquez G. 1998. Endemic phytotaxa of the Peninsula of Yucatán. Harvard Papers in Botany 3:263-314.

Eriksson O. 1989. Seedlings dynamics and life histories in clonal plants. Oikos 55:231-238.

Eriksson O. 1993. Dynamics of genets in clonal plants. Trends in Ecology \& Evolution 8:313-316.

Espadas-Manrique C., Durán R. y Argáez J. 2003. Phytogeographic analysis of taxa endemic to the Yucatán Peninsula using geographic information systems, the domain heuristic method and parsimony analysis of endemicity. Diversity and Distributions 9:313-330.

Esparza-Olguín L., Valverde T. y Vilchis-Anaya E. 2002. Demographic analysis of a rare columnar cactus (Neobuxbaumia macrocephala) in the Tehuacan Valley, Mexico. Biological Conservation 103:349-359.

Franco A.C. y Nobel P.S. 1989. Effect of nurse plant on the microhabitat and growth of cacti. Journal of Ecology 77:870-886.

García-Franco J.G. y Rico-Gray V. 1995. Population structure and clonal growth in Bromelia penguin L. (Bromeliaceae) in dry 
forest of coastal Veracruz, México. Tulane Studies in Zoology and Botany 30:27-37.

Godínez-Álvarez H., Valiente-Banuet A. y Valiente-Banuet L. 1999. Biotic interactions and the population dynamics of the longlived columnar cactus Neobuxbaumia tetetzo in the Tehuacan Valley, Mexico. Canadian Journal of Botany 77:203-208.

Godínez-Álvarez H., Valverde T. y Ortega-Baes P. 2003. Demographic trends in the Cactaceae. The Botanical Review 69:173-203.

Grime J.P. 1982. Estrategias de Adaptación de las Plantas y Procesos que Controlan la Vegetación. Limusa, México, D.F.

Guzmán U., Arias S. y Dávila P. 2003. Catálogo de Cactáceas Mexicanas. Universidad Nacional Autónoma de México-Comisión Nacional para el Conocimiento y Uso de la Biodiversidad, México, D.F.

Harada Y., Kawano S. e Iwasa Y. 1997. Probability of clonal identity: inferring the relative success of sexual versus clonal reproduction from spatial genetics patterns. Journal of Ecology 85:591-600.

Hartnett D.C. y Bazzaz F.A. 1983. Physiological integration among intraclonal ramets in Solidago canadensis. Ecology 64:779-788.

Harper J.L. 1977. Population Biology of Plants. Academic Press, Londres.

Harper J.L. y White J. 1974. The demography of plants. Annual Review of Ecological and Systematic 5:419-463.

IUCN [International Union for Conservation of Nature]. 1985. Rare, threatened and insufficiently known endemic cacti of Mexico endemic taxa. Threatened Plants Comittee, Botanic Gardens Conservation Coordinating Body. IUCN, Gland, Suiza.

Jordan P.W. y Nobel P.S. 1979. Infrequent establishment of seedlings of Agave deserti (Agavaceae) in the Northwestern Sonoran Desert. American Journal of Botany 66:1079-1084.

Klimeš L., Klimešová J., Hendricks R.J.J. y van Groenendael J.M. 1997. Clonal plant architecture: a comparative analysis of form and fuction. En: de Kroon H. y van Groenendael J. Eds. The Ecology and Evolution of Clonal Plant, pp. 1-29, Backhuys Publishers, Leiden.

Lefkovitch L. 1965. The study of population growth in organisms grouped by stages. Biometrics 21:1-18.

López-Haas A.L. 2007. Germinación, establecimiento y supervivencia de Mammillaria gaumeri, cactácea rara y endémica de la península de Yucatán. Tesis de Licenciatura, Instituto Tecnológico Agropecuario No. 2, Conkal, 76 pp.

López-Jiménez L. 2001. Distribución, abundancia y estructura poblacional de Mammillaria gaumeri (Britton \& Rose) Orcutt, especie rara y endémica de Yucatán. Tesis de Licenciatura, Instituto Tecnológico Agropecuario No. 2, Conkal, 70 pp.

Mandujano M.C., Montaña C. y Eguiarte L.E. 1996. Reproductive ecology and inbreeding depression in Opuntia rastrera (Cactaceae) in the Chihuahuan Desert. Why are sexually derived recruitments so rare? American Journal of Botany 83:63-70.

Mandujano M.C., Montaña C., Méndez I. y Golubov J. 1998. The relative contributions of sexual reproduction and clonal propagation in Opuntia rastrera from two habitats in the Chihuahuan Desert. Journal of Ecology 86:911-921.

Mandujano M.C., Montaña C., Franco M., Golubov J. y FloresMartínez A. 2001. Integration of demographic annual variability in a clonal desert cactus. Ecology 82:344-359.

Mandujano M.C., Verhulst-Johannes A.M., Carrillo-Ángeles I.G., y Golubov J. 2007. Population dynamics of Ariocarpus scaphi- rostris Bödeker (Cactaceae): Evaluating the status of a threatened species. International Journal Plant Science 168:10351044.

Mandujano-Sánchez M.C. 2007. La clonalidad y sus efectos en biología de poblaciones. En: Eguiarte L.E., Souza V. y Aguirre X. Comps. Ecología Molecular, pp.217-250, Secretaría de Medio Ambiente y Recursos Naturales-Instituto Nacional de Ecología-Universidad Nacional Autónoma de México-Comisión Nacional para el Conocimiento y Uso de la Biodiversidad, México, D.F.

McAuliffe J.R. 1984. Sahuaro-nurse tree associations in the Sonoran Desert: Competitive effects of sahuaros. Oecologia 64:319-321.

McFadden C.S. 1991. A comparative demographic analysis of clonal reproduction in a temperate soft coral. Ecology 72:1849-1866.

Méndez M., Durán R., Olmsted I. y Oyama K. 2004. Population dynamics of Pterocereus gaumeri, a rare and endemic columnar cactus of Mexico. Biotropica 36:492-504.

Mendoza-Ochoa A.E. 1994. Demografía e integración clonal en Reinhardtia gracilis, una palma tropical. Tesis doctoral, Facultad de Ciencias, Universidad Nacional Autónoma de México, México, D.F. 156 pp.

Miranda F. y Hernández-X. E. 1963. Los tipos de vegetación de México y su clasificación. Boletín de la Sociedad Botánica de México 28:29-179.

Mondragón D., Durán R., Ramírez I. y Valverde T. 2004. Temporal variation in the demography of the clonal epiphyte Tillandsia brachycaulos (Bromeliaceae) in the Yucatan Peninsula, Mexico. Journal of Tropical Ecology 20:189-200.

Nobel P.S.1988. Environmental Biology of Agaves and Cacti. Cambridge University Press, Cambridge.

Orellana R., Balam-Ku M., Bañuelos I., García M.E., GonzálezIturbe J.A., Herrera C.F. y Vidal L.J. 1999. Evaluación climática. En: García de Fuentes A. y Córdoba-Ordoñez J. Eds. Atlas de Procesos Territoriales de Yucatán, pp.163-182, Universidad Autónoma de Yucatán, Mérida.

Parker C.K. y Hamrick J.L. 1992. Genetic diversity and clonal structure in a columnar cactus, Lophocereus schottii. American Journal of Botany 79:86-96.

Pierson E.A. y Turner R.M. 1998. An 85-year study of saguaro (Carnegiea gigantea) demography. Ecology 79:2676-2693.

Schmalzel R.J., Reichenbacher F.W. y Rutman S. 1995. Demographic study of the rare Coryphantha robbinsorum (Cactaceae) in southeastern Arizona. Madroño 42:332-348.

Schmid B. y Bazzaz F.A. 1987. Clonal integration and population structure in perennials: effects of severing rhizome connections. Ecology 68:2016-2022.

SEMARNAT [Secretaria del Medio Ambiente y Recursos Naturales]. 2010. Norma Oficial Mexicana NOM-059-SEMARNAT-2010, Protección ambiental - Especies nativas de México de Flora y Fauna Silvestres - Categorías de Riesgo y especificaciones para su inclusión, exclusión o cambio - Lista de Especies en Riesgo. Diario Oficial de la Federación 2a Sección, 30 de diciembre del 2010. <http://www.semarnat.gob. mx/leyesynormas/SEMARNAT\%20DOF/Norma\%20Oficial\%20Mexicana\%20NOM-059-SEMARNAT-2010.pdf>

Silvertown J.W. y Lovett-Doust J. 1993. Introduction to Plant Population Biology. Blackwell Science, Oxford.

Silvertown J.W., Franco M., Pisanty I. y Mendoza A. 1993. Comparative plant demography: Relative importance of life-cycle components to the finite rate of increase in woody and herba- 
ceous perennials. Journal of Ecology 81:465-476.

Steenbergh W.F. y Lowe C.H. 1969. Critical factors during the first years of life of the saguaro (Cereus giganteus) at Saguaro National Monument, Arizona. Ecology 50:825-834.

Thien L.B., Bradburn A.S., Welden A.L. 1982. The Woody Vegetation of Dizbilchaltun, a Maya Archaeological Site in Northwest Yucatan, Mexico. Middle American Research Institute, Tulane University, Nueva Orleans.

Torres W., Méndez M., Dorantes A. y Durán R. 2010. Estructura, composición y diversidad del matorral de duna costera en el litoral yucateco. Boletín de la Sociedad Botánica de México 86:37-51.

Turner R.M., Alcorn S.M. y Olin G. 1969. Mortality of transplanted saguaro seedlings. Ecology 50:835-844.

Valiente-Banuet A. y Ezcurra E. 1991. Shade as a cause of the association between the cactus Neobuxbaumia tetetzo and the nurse plant Mimosa luisiana in the Tehuacan valley, Mexico. Journal of Ecology 79:961-971.

Valiente-Banuet A., Vite G. y Zavala-Hurtado A. 1991. Interaction between the cactus Neobuxbaumia tetetzo and the nurse shrubs Mimosa luisiana. Journal of Vegetation Science 2:12-14.

Valverde T., Quijas S., López-Villavicencio M. y Castillo S. 2004. Population dynamics of Mammillaria magnimamma Haworth. (Cactaceae) in a lava-field in central Mexico. Plant Ecology 170:167-184.

Valverde T., Trejo M.L. y Castillo S. 1999. Patrón de distribución y abundancia de Mammillaria magnimamma en la reserva del pedregal de San Ángel, México D.F. Cactáceas y Suculentas Mexicanas 44:64-74.

Zar H. 1984. Biostatistical Analysis. Prentice-Hall Inc., Englewood Clifffs.

Recibido: 28 de agosto de 2010

Aceptado: 31 de mayo de 2011 
Merari Ferrer, Rafael Durán, Martha Méndez, Alfredo Dorantes y Gabriel Dzib.

Apéndice 1. Matrices de transición de las poblaciones de Mammillaria gaumeri con sus tasas de crecimiento poblacional y sus intervalos de confianza. La fecundidad vía semillas está ubicada en el primer renglón, la fecundidad vía hijuelos se observa en la fila siete (negritas e itálicas). En negritas se observa la permanencia en la diagonal principal. w = estructura estable de edades, $\mathrm{v}=$ valor reproductivo, $\mathrm{N}=$ número de individuos por categoría.

\begin{tabular}{|c|c|c|c|c|c|c|c|c|c|c|c|c|}
\hline \multicolumn{4}{|c|}{ Chuburná 2004-2005 } & \multicolumn{9}{|c|}{$\lambda=1.0041 \pm 0.114$} \\
\hline & $\mathbf{P}$ & I & J & Al & All & AlII & ic & jc & ac1 & ac2 & w & $\mathrm{v}$ \\
\hline $\mathbf{P}$ & 0.0000 & 0.0000 & 0.0000 & 0.0000 & 7.5690 & 4.4080 & 0.0000 & 0.0000 & 0.0000 & 3.4800 & 0.8210 & 0.0000 \\
\hline I & 0.0010 & 0.0010 & 0.0000 & 0.0000 & 0.0000 & 0.0000 & 0.0000 & 0.0000 & 0.0000 & 0.0000 & 0.0390 & 0.0000 \\
\hline J & 0.0000 & 0.0010 & 0.0010 & 0.0000 & 0.0000 & 0.0000 & 0.0000 & 0.0000 & 0.0000 & 0.0000 & 0.0000 & 0.0000 \\
\hline Al & 0.0000 & 0.0000 & 0.0010 & 0.0010 & 0.0000 & 0.0000 & 0.0000 & 0.0000 & 0.0000 & 0.0000 & 0.0000 & 0.0000 \\
\hline All & 0.0000 & 0.0000 & 0.0000 & 0.0010 & 0.8750 & 0.1110 & 0.0000 & 0.0000 & 0.0000 & 0.0000 & 0.0660 & 0.2650 \\
\hline AlII & 0.0000 & 0.0000 & 0.0000 & 0.0000 & 0.1250 & 0.8890 & 0.0000 & 0.0000 & 0.0000 & 0.0000 & 0.0740 & 0.2650 \\
\hline ic & 0.0000 & 0.0000 & 0.0000 & 0.0000 & 0.0000 & 0.0000 & 0.0010 & 0.0000 & 0.0000 & 0.0000 & 0.0000 & 0.0000 \\
\hline jc & 0.0000 & 0.0000 & 0.0000 & 0.0000 & 0.0000 & 0.0000 & 0.0010 & 0.4000 & 0.0000 & 0.0000 & 0.0000 & 0.1560 \\
\hline ac1 & 0.0000 & 0.0000 & 0.0000 & 0.0000 & 0.0000 & 0.0000 & 0.0000 & 0.6000 & 0.5000 & 0.0000 & 0.0000 & 0.1560 \\
\hline ac2 & 0.0000 & 0.0000 & 0.0000 & 0.0000 & 0.0000 & 0.0000 & 0.0000 & 0.0000 & 0.5000 & 1.0000 & 0.0000 & 0.1560 \\
\hline $\mathrm{N}$ & 62 & 10 & 10 & 10 & 8 & 9 & 10 & 5 & 4 & 6 & & \\
\hline
\end{tabular}

\begin{tabular}{|c|c|c|c|c|c|c|c|c|c|c|c|c|}
\hline \multicolumn{4}{|c|}{ Chuburná 2005-2006 } & \multicolumn{9}{|c|}{$\lambda=1.0007 \pm 0.117$} \\
\hline & $\mathbf{P}$ & I & J & Al & AlI & AlII & ic & jc & ac1 & ac2 & w & $\mathbf{v}$ \\
\hline $\mathbf{P}$ & 0.0000 & 0.0000 & 0.0000 & 3.1680 & 2.8710 & 1.8810 & 0.0000 & 0.0000 & 0.0000 & 0.9900 & 0.3590 & 0.0000 \\
\hline I & 0.0160 & 0.0010 & 0.0000 & 0.0000 & 0.0000 & 0.0000 & 0.0000 & 0.0000 & 0.0000 & 0.0000 & 0.0060 & 0.0000 \\
\hline J & 0.0000 & 0.0010 & 0.0010 & 0.0000 & 0.0000 & 0.0000 & 0.0000 & 0.0000 & 0.0000 & 0.0000 & 0.0000 & 0.0000 \\
\hline Al & 0.0000 & 0.0000 & 0.0010 & 0.1000 & 0.0000 & 0.0000 & 0.0000 & 0.0000 & 0.0000 & 0.0000 & 0.0000 & 0.0000 \\
\hline All & 0.0000 & 0.0000 & 0.0000 & 0.9000 & 0.3750 & 0.0000 & 0.0000 & 0.0000 & 0.0000 & 0.0000 & 0.0000 & 0.0000 \\
\hline AIII & 0.0000 & 0.0000 & 0.0000 & 0.0000 & 0.2500 & 0.3750 & 0.0000 & 0.0000 & 0.0000 & 0.0000 & 0.0000 & 0.0000 \\
\hline ic & 0.0000 & 0.0000 & 0.0000 & 1.0000 & 0.0000 & 0.2500 & 0.0010 & 0.0000 & 0.0000 & 0.0000 & 0.0000 & 0.0000 \\
\hline jc & 0.0000 & 0.0000 & 0.0000 & 0.0000 & 0.0000 & 0.0000 & 0.0010 & 0.7500 & 0.0000 & 0.0000 & 0.0000 & 0.2000 \\
\hline ac1 & 0.0000 & 0.0000 & 0.0000 & 0.0000 & 0.0000 & 0.0000 & 0.0000 & 0.1250 & 0.8330 & 0.1250 & 0.2720 & 0.4000 \\
\hline ac2 & 0.0000 & 0.0000 & 0.0000 & 0.0000 & 0.0000 & 0.0000 & 0.0000 & 0.0000 & 0.1670 & 0.8750 & 0.3630 & 0.4000 \\
\hline $\mathrm{N}$ & 3 & 10 & 10 & 10 & 8 & 8 & 10 & 8 & 6 & 8 & & \\
\hline
\end{tabular}

\begin{tabular}{|c|c|c|c|c|c|c|c|c|c|c|c|c|}
\hline \multicolumn{4}{|c|}{ Chuburná 2006-2007 } & \multicolumn{9}{|c|}{$\lambda=1.0116 \pm 0.003$} \\
\hline & $\mathbf{P}$ & I & $J$ & $\mathrm{Al}$ & AlI & AlII & ic & jc & ac1 & ac2 & $\mathbf{w}$ & $\mathbf{v}$ \\
\hline $\mathbf{P}$ & 0.0000 & 0.0000 & 0.0000 & 5.5010 & 13.294 & 5.2260 & 0.0000 & 0.0000 & 0.0000 & 1.9650 & 0.16276 & 0.00000 \\
\hline I & 0.0010 & 0.0010 & 0.0000 & 0.0000 & 0.0000 & 0.0000 & 0.0000 & 0.0000 & 0.0000 & 0.0000 & 0.00016 & 0.00000 \\
\hline J & 0.0000 & 0.0010 & 0.0010 & 0.00000 & 0.0000 & 0.0000 & 0.0000 & 0.0000 & 0.0000 & 0.0000 & 0.00000 & 0.00033 \\
\hline $\mathrm{Al}$ & 0.0000 & 0.0000 & 0.0010 & 0.9000 & 0.0000 & 0.0000 & 0.0000 & 0.0000 & 0.00000 & 0.0000 & 0.00000 & 0.33322 \\
\hline All & 0.0000 & 0.0000 & 0.0000 & 0.1000 & 0.3330 & 0.0000 & 0.0000 & 0.0000 & 0.0000 & 0.0000 & 0.00000 & 0.33322 \\
\hline AllI & 0.0000 & 0.0000 & 0.0000 & 0.0000 & 0.6670 & 1.0000 & 0.0000 & 0.0000 & 0.0000 & 0.0000 & 0.01204 & 0.33322 \\
\hline ic & 0.0000 & 0.0000 & 0.0000 & 1.0000 & 1.6670 & 0.6000 & 0.9900 & 0.0000 & 0.0000 & 0.0000 & 0.72229 & 0.00000 \\
\hline jc & 0.0000 & 0.0000 & 0.0000 & 0.0000 & 0.0000 & 0.0000 & 0.0100 & 0.1430 & 0.0000 & 0.0000 & 0.00843 & 0.00000 \\
\hline ac1 & 0.0000 & 0.0000 & 0.0000 & 0.0000 & 0.0000 & 0.0000 & 0.0000 & 0.8570 & 0.6670 & 0.1430 & 0.04351 & 0.00000 \\
\hline ac2 & 0.0000 & 0.0000 & 0.0000 & 0.0000 & 0.0000 & 0.0000 & 0.0000 & 0.0000 & 0.1670 & 0.8570 & 0.05081 & 0.00000 \\
\hline $\mathrm{N}$ & 525 & 10 & 10 & 10 & 3 & 5 & 5 & 7 & 6 & 7 & & \\
\hline
\end{tabular}


Apéndice 1. Continuación

\begin{tabular}{|c|c|c|c|c|c|c|c|c|c|c|c|c|}
\hline \multicolumn{3}{|c|}{ Aruba 2004-2005 } & \multicolumn{10}{|c|}{$\lambda=1.0544 \pm 0.137$} \\
\hline & $\mathbf{P}$ & I & $J$ & Al & AlI & AIII & ic & jc & ac1 & ac2 & w & $\mathrm{v}$ \\
\hline $\mathbf{P}$ & 0.0000 & 0.0000 & 0.0000 & 0.5690 & 4.3250 & 12.528 & 0.0000 & 0.0000 & 0.7460 & 4.5240 & 0.5929 & 0.0034 \\
\hline I & 0.0010 & 0.9000 & 0.0000 & 0.0000 & 0.0000 & 0.0000 & 0.0000 & 0.0000 & 0.0000 & 0.0000 & 0.1872 & 0.0734 \\
\hline$J$ & 0.0000 & 0.10000 & 0.7140 & 0.0910 & 0.0000 & 0.0000 & 0.0000 & 0.0000 & 0.0000 & 0.0000 & 0.0716 & 0.1115 \\
\hline Al & 0.0000 & 0.0000 & 0.2860 & 0.6820 & 0.0360 & 0.0000 & 0.0000 & 0.0000 & 0.0000 & 0.0000 & 0.0604 & 0.1318 \\
\hline All & 0.0000 & 0.0000 & 0.0000 & 0.2270 & 0.7860 & 0.0000 & 0.0000 & 0.0000 & 0.0000 & 0.0000 & 0.0515 & 0.1618 \\
\hline AlII & 0.0000 & 0.0000 & 0.0000 & 0.0000 & 0.1430 & 0.8000 & 0.0000 & 0.0000 & 0.0000 & 0.0000 & 0.0292 & 0.1664 \\
\hline ic & 0.0000 & 0.0000 & 0.0000 & 0.0000 & 0.1430 & 0.0000 & 0.0010 & 0.0000 & 0.0000 & 0.0000 & 0.0070 & $9 \mathrm{E}-05$ \\
\hline jc & 0.0000 & 0.0000 & 0.0000 & 0.0000 & 0.0000 & 0.0000 & 0.0010 & 0.6670 & 0.0000 & 0.0000 & $2 \mathrm{E}-05$ & 0.0930 \\
\hline ac1 & 0.0000 & 0.0000 & 0.0000 & 0.0000 & 0.0000 & 0.0000 & 0.0000 & 0.3330 & 0.9290 & 0.1670 & $9 \mathrm{E}-05$ & 0.1075 \\
\hline ac2 & 0.0000 & 0.0000 & 0.0000 & 0.0000 & 0.0000 & 0.0000 & 0.0000 & 0.0000 & 0.0710 & 0.8330 & $3 \mathrm{E}-05$ & 0.1511 \\
\hline $\mathrm{N}$ & 62 & 10 & 14 & 22 & 28 & 5 & 10 & 9 & 14 & 6 & & \\
\hline \multicolumn{3}{|c|}{ Aruba 2005-2006 } & \multicolumn{10}{|c|}{$\lambda=0.8483 \pm 0.158$} \\
\hline & $\mathbf{P}$ & I & $J$ & Al & AlI & AIII & ic & jc & ac1 & ac2 & w & $\mathbf{v}$ \\
\hline $\mathbf{P}$ & 0.0000 & 0.00000 & 0.0000 & 0.2380 & 1.7010 & 2.9700 & 0.0000 & 0.0000 & 0.2330 & 1.7160 & 0.3867 & 0.0005 \\
\hline I & 0.0160 & 0.1000 & 0.0000 & 0.0000 & 0.0000 & 0.0000 & 0.0000 & 0.0000 & 0.0000 & 0.0000 & 0.0082 & 0.0240 \\
\hline$J$ & 0.0000 & 0.1000 & 0.5000 & 0.0000 & 0.0000 & 0.0000 & 0.0000 & 0.0000 & 0.0000 & 0.0000 & 0.0024 & 0.1804 \\
\hline Al & 0.0000 & 0.0000 & 0.0830 & 0.8500 & 0.0000 & 0.0000 & 0.0000 & 0.0000 & 0.0000 & 0.0000 & 0.3829 & 0.7617 \\
\hline All & 0.0000 & 0.0000 & 0.0000 & 0.0500 & 0.5560 & 0.1250 & 0.0000 & 0.0000 & 0.0000 & 0.0000 & 0.0771 & 0.0056 \\
\hline AIII & 0.0000 & 0.0000 & 0.0000 & 0.0000 & 0.0370 & 0.7500 & 0.0000 & 0.0000 & 0.0000 & 0.0000 & 0.0284 & 0.0203 \\
\hline ic & 0.0000 & 0.0000 & 0.0000 & 0.0000 & 0.1110 & 0.0000 & 0.1000 & 0.0000 & 0.0000 & 0.0000 & 0.0114 & 0.0012 \\
\hline jc & 0.0000 & 0.0000 & 0.0000 & 0.0000 & 0.0000 & 0.0000 & 0.9000 & 0.6670 & 0.0590 & 0.0000 & 0.0651 & 0.0010 \\
\hline ac1 & 0.0000 & 0.0000 & 0.0000 & 0.0000 & 0.0000 & 0.0000 & 0.0000 & 0.1670 & 0.4710 & 0.0000 & 0.0287 & 0.0011 \\
\hline ac2 & 0.0000 & 0.0000 & 0.0000 & 0.0000 & 0.0000 & 0.0000 & 0.0000 & 0.0000 & 0.0590 & 0.6670 & 0.0092 & 0.0042 \\
\hline $\mathrm{N}$ & 6 & 10 & 12 & 20 & 27 & 8 & 10 & 6 & 17 & 6 & & \\
\hline \multicolumn{3}{|c|}{ Aruba 2006-2007 } & \multicolumn{10}{|c|}{$\lambda=1.0147 \pm 0.014$} \\
\hline & $\mathbf{P}$ & I & $J$ & Al & AII & AIII & ic & jc & ac1 & ac2 & w & $\mathbf{v}$ \\
\hline $\mathbf{P}$ & 0.0000 & 0.0000 & 0.0000 & 0.4580 & 4.9850 & 5.8940 & 0.0000 & 0.0000 & 0.7640 & 3.5760 & 0.7789 & 0.0000 \\
\hline I & 0.0010 & 0.0010 & 0.0000 & 0.0000 & 0.0000 & 0.0000 & 0.0000 & 0.0000 & 0.0000 & 0.0000 & 0.0008 & 0.0002 \\
\hline$J$ & 0.0000 & 0.0010 & 0.8570 & 0.0000 & 0.0000 & 0.0000 & 0.0000 & 0.0000 & 0.0000 & 0.0000 & $1 \mathrm{E}-05$ & 0.1992 \\
\hline Al & 0.0000 & 0.0000 & 0.1430 & 0.7780 & 0.0000 & 0.0000 & 0.0000 & 0.0000 & 0.0000 & 0.0000 & 0.0000 & 0.1998 \\
\hline All & 0.0000 & 0.0000 & 0.0000 & 0.1670 & 0.6880 & 0.0000 & 0.0000 & 0.0000 & 0.0000 & 0.0000 & 0.0000 & 0.2662 \\
\hline AIII & 0.0000 & 0.0000 & 0.0000 & 0.0000 & 0.2500 & 1.0000 & 0.0000 & 0.0000 & 0.0000 & 0.0000 & 0.0010 & 0.3326 \\
\hline ic & 0.0000 & 0.0000 & 0.0000 & 0.0560 & 0.0630 & 0.8570 & 0.1000 & 0.0000 & 0.0000 & 0.0000 & 0.0009 & 0.0002 \\
\hline jc & 0.0000 & 0.0000 & 0.0000 & 0.0000 & 0.0000 & 0.0000 & 0.9000 & 0.3850 & 0.0000 & 0.0000 & 0.0014 & 0.0002 \\
\hline ac1 & 0.0000 & 0.0000 & 0.0000 & 0.0000 & 0.0000 & 0.0000 & 0.0000 & 0.5380 & 0.2220 & 0.0000 & 0.0009 & 0.0002 \\
\hline ac2 & 0.0000 & 0.0000 & 0.0000 & 0.0000 & 0.0000 & 0.0000 & 0.0000 & 0.0000 & 0.1110 & 1.0000 & 0.2161 & 0.0015 \\
\hline $\mathrm{N}$ & 525 & 10 & 7 & 18 & 16 & 7 & 10 & 13 & 9 & 5 & & \\
\hline
\end{tabular}


Merari Ferrer, Rafael Durán, Martha Méndez, Alfredo Dorantes y Gabriel Dzib.

Apéndice 1. Continuación

\begin{tabular}{|c|c|c|c|c|c|c|c|c|c|c|c|c|}
\hline \multicolumn{3}{|c|}{ Chicxulub 2004-2005 } & \multicolumn{10}{|c|}{$\lambda=1.2281 \pm 0.127$} \\
\hline & $\mathbf{P}$ & I & J & Al & All & AlII & ic & jc & ac1 & ac2 & w & $\mathrm{v}$ \\
\hline $\mathbf{P}$ & 0.0000 & 0.0000 & 0.0000 & 3.863 & 15.138 & 75.168 & 0.0000 & 0.0000 & 0.0000 & 0.0000 & 0.8405 & 0.0018 \\
\hline I & 0.0480 & 0.3330 & 0.0000 & 0.0000 & 0.0000 & 0.0000 & 0.0000 & 0.0000 & 0.0000 & 0.0000 & 0.0452 & 0.0458 \\
\hline J & 0.0000 & 0.6670 & 0.7000 & 0.0000 & 0.0000 & 0.0000 & 0.0000 & 0.0000 & 0.0000 & 0.0000 & 0.0573 & 0.0613 \\
\hline Al & 0.0000 & 0.0000 & 0.3000 & 0.5500 & 0.0000 & 0.0000 & 0.0000 & 0.0000 & 0.0000 & 0.0000 & 0.0254 & 0.1076 \\
\hline All & 0.0000 & 0.0000 & 0.0000 & 0.3500 & 0.8570 & 0.0000 & 0.0000 & 0.0000 & 0.0000 & 0.0000 & 0.0241 & 0.1880 \\
\hline AllI & 0.0000 & 0.0000 & 0.0000 & 0.0000 & 0.0710 & 1.0000 & 0.0000 & 0.0000 & 0.0000 & 0.0000 & 0.0076 & 0.5956 \\
\hline ic & 0.0000 & 0.0000 & 0.0000 & 0.0000 & 0.0000 & 0.0000 & 0.8240 & 0.0000 & 0.0000 & 0.0000 & 0.0000 & 0.0000 \\
\hline jc & 0.0000 & 0.0000 & 0.0000 & 0.0000 & 0.0000 & 0.0000 & 0.1760 & 0.5000 & 0.0000 & 0.0000 & 0.0000 & 0.0000 \\
\hline ac1 & 0.0000 & 0.0000 & 0.0000 & 0.0000 & 0.0000 & 0.0000 & 0.0000 & 0.5000 & 0.7500 & 0.0000 & 0.0000 & 0.0000 \\
\hline ac2 & 0.0000 & 0.0000 & 0.0000 & 0.0000 & 0.0000 & 0.0000 & 0.0000 & 0.0000 & 0.2500 & 0.1660 & 0.0000 & 0.0000 \\
\hline $\mathrm{N}$ & 62 & 3 & 20 & 20 & 28 & 10 & 17 & 8 & 4 & 6 & & \\
\hline \multicolumn{3}{|c|}{ Chicxulub 2005-2006 } & \multicolumn{10}{|c|}{$\lambda=0.9984 \pm 0.132$} \\
\hline & $\mathbf{P}$ & $I$ & $J$ & Al & All & AIII & ic & jc & ac1 & ac2 & w & $\mathrm{v}$ \\
\hline $\mathbf{P}$ & 0.0000 & 0.0000 & 0.0000 & 1.724 & 5.186 & 9.504 & 0.0000 & 0.0000 & 0.0000 & 0.0000 & 0.1420 & 0.0032 \\
\hline I & 0.0160 & 0.1000 & 0.0620 & 0.0000 & 0.0000 & 0.0000 & 0.0000 & 0.0000 & 0.0000 & 0.0000 & 0.0038 & 0.1970 \\
\hline J & 0.0000 & 0.900 & 0.8130 & 0.0000 & 0.0000 & 0.0000 & 0.0000 & 0.0000 & 0.0000 & 0.0000 & 0.0184 & 0.1968 \\
\hline Al & 0.0000 & 0.0000 & 0.1250 & 0.8240 & 0.1300 & 0.0000 & 0.0000 & 0.0000 & 0.0000 & 0.0000 & 0.0259 & 0.1942 \\
\hline All & 0.0000 & 0.0000 & 0.0000 & 0.1180 & 0.8060 & 0.3330 & 0.0000 & 0.0000 & 0.0000 & 0.0000 & 0.0172 & 0.2426 \\
\hline AllI & 0.0000 & 0.0000 & 0.0000 & 0.0000 & 0.0320 & 0.3330 & 0.0000 & 0.0000 & 0.0000 & 0.0000 & 0.0008 & 0.1662 \\
\hline ic & 0.0000 & 0.0000 & 0.0000 & 0.0000 & 0.0650 & 0.0000 & 0.7150 & 0.0000 & 0.0000 & 0.0000 & 0.0039 & 0.0000 \\
\hline jc & 0.0000 & 0.0000 & 0.0000 & 0.0000 & 0.0000 & 0.0000 & 0.0710 & 0.4440 & 0.0000 & 0.0000 & 0.0005 & 0.0000 \\
\hline ac1 & 0.0000 & 0.0000 & 0.0000 & 0.0000 & 0.0000 & 0.0000 & 0.0000 & 0.2220 & 0.8330 & 0.5000 & 0.5909 & 0.0000 \\
\hline ac2 & 0.0000 & 0.0000 & 0.0000 & 0.0000 & 0.0000 & 0.0000 & 0.0000 & 0.0000 & 0.1660 & 0.5000 & 0.1964 & 0.0000 \\
\hline N & 6 & 10 & 16 & 17 & 31 & 3 & 14 & 9 & 8 & 2 & & \\
\hline \multicolumn{3}{|c|}{ Chicxulub 2006-2007 } & \multicolumn{10}{|c|}{$\lambda=0.9946 \pm 0.208$} \\
\hline & $\mathbf{P}$ & $I$ & $J$ & Al & All & AIII & ic & jc & ac1 & ac2 & w & v \\
\hline $\mathbf{P}$ & 0.0000 & 0.0000 & 0.0000 & 2.4230 & 9.9700 & 49.507 & 0.0000 & 0.0000 & 0.0000 & 0.0000 & 0.9523 & 0.0000 \\
\hline I & 0.0010 & 0.0010 & 0.0000 & 0.0000 & 0.0000 & 0.0000 & 0.0000 & 0.0000 & 0.0000 & 0.0000 & 0.0011 & 0.0004 \\
\hline J & 0.0000 & 0.0010 & 0.6430 & 0.0000 & 0.0000 & 0.0000 & 0.0000 & 0.0000 & 0.0000 & 0.0000 & 0.0000 & 0.3901 \\
\hline Al & 0.0000 & 0.0000 & 0.3570 & 0.4760 & 0.0000 & 0.0000 & 0.0000 & 0.0000 & 0.0000 & 0.0000 & 0.0000 & 0.2811 \\
\hline All & 0.0000 & 0.0000 & 0.0000 & 0.5240 & 0.8210 & 0.0000 & 0.0000 & 0.0000 & 0.0000 & 0.0000 & $2 \mathrm{E}-05$ & 0.2276 \\
\hline AllI & 0.0000 & 0.0000 & 0.0000 & 0.0000 & 0.1790 & 0.9000 & 0.0000 & 0.0000 & 0.0000 & 0.0000 & 0.0173 & 0.1007 \\
\hline ic & 0.0000 & 0.0000 & 0.0000 & 0.2860 & 0.0950 & 0.1430 & 0.6000 & 0.0000 & 0.0000 & 0.0000 & $2 \mathrm{E}-05$ & 0.0000 \\
\hline jc & 0.0000 & 0.0000 & 0.0000 & 0.0000 & 0.0000 & 0.0000 & 0.2000 & 0.4000 & 0.0000 & 0.0000 & $1 \mathrm{E}-05$ & 0.0000 \\
\hline ac1 & 0.0000 & 0.0000 & 0.0000 & 0.0000 & 0.0000 & 0.0000 & 0.0000 & 0.6000 & 0.7780 & 0.0000 & $3 \mathrm{E}-05$ & 0.0000 \\
\hline ac2 & 0.0000 & 0.0000 & 0.0000 & 0.0000 & 0.0000 & 0.0000 & 0.0000 & 0.0000 & 0.2220 & 0.9000 & 0.0293 & 0.0000 \\
\hline $\mathrm{N}$ & 525 & 10 & 14 & 21 & 28 & 10 & 15 & 10 & 9 & 10 & & \\
\hline
\end{tabular}


Apéndice 1. Continuación

\begin{tabular}{|c|c|c|c|c|c|c|c|c|c|c|c|c|}
\hline \multicolumn{4}{|c|}{ Uaymitún 2004-2005 } & \multicolumn{9}{|c|}{$\lambda=1.0163 \pm 0.006$} \\
\hline & $\mathbf{P}$ & I & J & Al & All & AIII & ic & jc & ac1 & ac2 & w & v \\
\hline $\mathbf{P}$ & 0.0000 & 0.0000 & 0.0000 & 14.152 & 13.920 & 45.240 & 0.0000 & 0.0000 & 0.7590 & 11.484 & 0.8975 & 0.0000 \\
\hline I & 0.0480 & 0.0010 & 0.0000 & 0.0000 & 0.0000 & 0.0000 & 0.0000 & 0.0000 & 0.0000 & 0.0000 & 0.0431 & 9E-05 \\
\hline$J$ & 0.0000 & 0.0010 & 0.1000 & 0.0000 & 0.0000 & 0.0000 & 0.0000 & 0.0000 & 0.0000 & 0.0000 & $4 \mathrm{E}-05$ & 0.0926 \\
\hline Al & 0.0000 & 0.0000 & 0.9000 & 0.8330 & 0.0830 & 0.0000 & 0.0000 & 0.0000 & 0.0000 & 0.0000 & 0.0003 & 0.0927 \\
\hline All & 0.0000 & 0.0000 & 0.0000 & 0.0560 & 0.8330 & 0.0000 & 0.0000 & 0.0000 & 0.0000 & 0.0000 & 0.0001 & 0.1995 \\
\hline AIII & 0.0000 & 0.0000 & 0.0000 & 0.0000 & 0.0420 & 1.0000 & 0.0000 & 0.0000 & 0.0000 & 0.0000 & 0.0066 & 0.3062 \\
\hline ic & 0.0000 & 0.0000 & 0.0000 & 0.0560 & 0.1670 & 0.0000 & 0.6670 & 0.0000 & 0.0000 & 0.0000 & 0.0001 & 0.0768 \\
\hline jc & 0.0000 & 0.0000 & 0.0000 & 0.0000 & 0.0000 & 0.0000 & 0.3330 & 0.9000 & 0.0000 & 0.0000 & 0.0003 & 0.0770 \\
\hline ac1 & 0.0000 & 0.0000 & 0.0000 & 0.0000 & 0.0000 & 0.0000 & 0.0000 & 0.1000 & 0.8180 & 0.0000 & 0.0002 & 0.0775 \\
\hline ac2 & 0.0000 & 0.0000 & 0.0000 & 0.0000 & 0.0000 & 0.0000 & 0.0000 & 0.0000 & 0.1820 & 1.0000 & 0.0518 & 0.0777 \\
\hline $\mathrm{N}$ & 62 & 10 & 10 & 18 & 24 & 3 & 3 & 10 & 11 & 4 & & \\
\hline \multicolumn{4}{|c|}{ Uaymitún 2005-2006 } & \multicolumn{9}{|c|}{$\lambda=1.0063 \pm 0.002$} \\
\hline & $\mathbf{P}$ & I & $J$ & $\mathrm{Al}$ & AII & AIII & ic & jc & ac1 & ac2 & w & $\mathbf{v}$ \\
\hline $\mathbf{P}$ & 0.0000 & 0.0000 & 0.0000 & 5.3680 & 6.0340 & 12.870 & 0.0000 & 0.0000 & 0.3520 & 2.9040 & 0.8175 & 0.0000 \\
\hline I & 0.0160 & 0.0100 & 0.0000 & 0.0000 & 0.0000 & 0.0000 & 0.0000 & 0.0000 & 0.0000 & 0.0000 & 0.0132 & $2 \mathrm{E}-05$ \\
\hline$J$ & 0.0000 & 0.0100 & 0.0100 & 0.0000 & 0.0000 & 0.0000 & 0.0000 & 0.0000 & 0.0000 & 0.0000 & 0.0001 & 0.0018 \\
\hline Al & 0.0000 & 0.0000 & 0.0100 & 0.6670 & 0.0470 & 0.0000 & 0.0000 & 0.0000 & 0.0000 & 0.0000 & $1 \mathrm{E}-05$ & 0.1737 \\
\hline All & 0.0000 & 0.0000 & 0.0000 & 0.1670 & 0.9050 & 0.0000 & 0.0000 & 0.0000 & 0.0000 & 0.0000 & $1 \mathrm{E}-05$ & 0.3463 \\
\hline AlII & 0.0000 & 0.0000 & 0.0000 & 0.0000 & 0.0480 & 1.0000 & 0.0000 & 0.0000 & 0.0000 & 0.0000 & 0.0328 & 0.2639 \\
\hline ic & 0.0000 & 0.0000 & 0.0000 & 0.0000 & 0.3330 & 0.0000 & 0.1670 & 0.0000 & 0.0000 & 0.0000 & 0.0000 & 0.0357 \\
\hline jc & 0.0000 & 0.0000 & 0.0000 & 0.0000 & 0.0000 & 0.0000 & 0.5000 & 0.8000 & 0.0000 & 0.0000 & $1 \mathrm{E}-05$ & 0.0595 \\
\hline ac1 & 0.0000 & 0.0000 & 0.0000 & 0.0000 & 0.0000 & 0.0000 & 0.0000 & 0.2000 & 0.8890 & 0.0000 & $2 \mathrm{E}-05$ & 0.0595 \\
\hline ac2 & 0.0000 & 0.0000 & 0.0000 & 0.0000 & 0.0000 & 0.0000 & 0.0000 & 0.0000 & 0.1110 & 1.0000 & 0.1364 & 0.0595 \\
\hline $\mathrm{N}$ & 6 & 10 & 10 & 18 & 21 & 4 & 6 & 10 & 9 & 6 & & \\
\hline \multicolumn{4}{|c|}{ Uaymitún 2006-2007 } & \multicolumn{9}{|c|}{$\lambda=0.9274 \pm 0.070$} \\
\hline & $\mathbf{P}$ & I & $J$ & Al & All & AlII & ic & jc & ac1 & ac2 & w & $\mathbf{v}$ \\
\hline $\mathbf{P}$ & 0.0000 & 0.0000 & 0.0000 & 12.906 & 10.001 & 17.878 & 0.0000 & 0.0000 & 0.5000 & 5.0420 & 0.8411 & 0.0000 \\
\hline I & 0.0010 & 0.0100 & 0.0000 & 0.0000 & 0.0000 & 0.0000 & 0.0000 & 0.0000 & 0.0000 & 0.0000 & 0.0009 & $4 \mathrm{E}-05$ \\
\hline$J$ & 0.0000 & 0.0100 & 0.0100 & 0.0000 & 0.0000 & 0.0000 & 0.0000 & 0.0000 & 0.0000 & 0.0000 & $1 \mathrm{E}-05$ & 0.004 \\
\hline Al & 0.0000 & 0.0000 & 0.0100 & 0.5380 & 0.0000 & 0.0000 & 0.0000 & 0.0000 & 0.0000 & 0.0000 & 0.0000 & 0.3632 \\
\hline AlI & 0.0000 & 0.0000 & 0.0000 & 0.3080 & 0.9100 & 0.2000 & 0.0000 & 0.0000 & 0.0000 & 0.0000 & 0.067 & 0.4588 \\
\hline AIII & 0.0000 & 0.0000 & 0.0000 & 0.0000 & 0.0450 & 0.4000 & 0.0000 & 0.0000 & 0.0000 & 0.0000 & 0.0057 & 0.1741 \\
\hline ic & 0.0000 & 0.0000 & 0.0000 & 0.0000 & 0.4090 & 0.0000 & 0.3750 & 0.0000 & 0.0000 & 0.0000 & 0.0496 & 0.0000 \\
\hline jc & 0.0000 & 0.0000 & 0.0000 & 0.0000 & 0.0000 & 0.0000 & 0.2500 & 0.3850 & 0.0000 & 0.0000 & 0.0229 & 0.0000 \\
\hline ac1 & 0.0000 & 0.0000 & 0.0000 & 0.0000 & 0.0000 & 0.0000 & 0.0000 & 0.3080 & 0.364 & 0.0000 & 0.0125 & 0.0000 \\
\hline ac2 & 0.0000 & 0.0000 & 0.0000 & 0.0000 & 0.0000 & 0.0000 & 0.0000 & 0.0000 & 0.091 & 0.5000 & 0.0003 & 0.0000 \\
\hline $\mathrm{N}$ & 525 & 10 & 10 & 13 & 22 & 5 & 8 & 13 & 11 & 6 & & \\
\hline
\end{tabular}


Merari Ferrer, Rafael Durán, Martha Méndez, Alfredo Dorantes y Gabriel Dzib.

Apéndice 1. Continuación

\begin{tabular}{lllllllllllll}
\hline \multicolumn{1}{l}{ San Benito 2004-2005 } & \multicolumn{1}{l}{$\boldsymbol{\lambda}=\mathbf{1 . 2 6 2 9} \pm \mathbf{0 . 2 6 2}$} \\
\hline & $\mathbf{P}$ & $\mathbf{I}$ & $\mathbf{J}$ & $\mathbf{A l}$ & All & All & ic & jc & ac1 & ac2 & $\mathbf{w}$ & $\mathbf{v}$ \\
$\mathbf{P}$ & $\mathbf{0 . 0 0 0 0}$ & 0.0000 & 0.0000 & 4.0840 & 23.444 & 18.643 & 0.0000 & 0.0000 & 3.1770 & 19.662 & 0.2258 & 0.0042 \\
$\mathbf{I}$ & 1.0000 & $\mathbf{0 . 9 0 0 0}$ & 0.0000 & 0.0000 & 0.0000 & 0.0000 & 0.0000 & 0.0000 & 0.0000 & 0.0000 & 0.6225 & 0.0053 \\
$\mathbf{J}$ & 0.0000 & 0.1000 & $\mathbf{0 . 7 0 0 0}$ & 0.0290 & 0.0000 & 0.0000 & 0.0000 & 0.0000 & 0.0000 & 0.0000 & 0.1117 & 0.0193 \\
AI & 0.0000 & 0.0000 & 0.1000 & $\mathbf{0 . 7 5 0 0}$ & 0.018 & 0.0000 & 0.0000 & 0.0000 & 0.0000 & 0.0000 & 0.0221 & 0.1085 \\
All & 0.0000 & 0.0000 & 0.0000 & 0.1180 & $\mathbf{0 . 9 1 2 0}$ & 0.4290 & 0.0000 & 0.0000 & 0.0000 & 0.0000 & 0.0079 & 0.3159 \\
AlII & 0.0000 & 0.0000 & 0.0000 & 0.0000 & 0.0350 & $\mathbf{0 . 5 0 0 0}$ & 0.0000 & 0.0000 & 0.0000 & 0.0000 & 0.0004 & 0.2819 \\
ic & 0.0000 & 0.0000 & 0.0000 & $\mathbf{0 . 1 1 8 0}$ & $\mathbf{0 . 0 7 0 0}$ & $\mathbf{0 . 2 1 4 0}$ & $\mathbf{0 . 5 8 8 0}$ & 0.0210 & 0.0000 & 0.0000 & 0.0049 & 0.0051 \\
jc & 0.0000 & 0.0000 & 0.0000 & 0.0000 & 0.0000 & 0.0000 & 0.2940 & $\mathbf{0 . 8 9 4 0}$ & 0.0430 & 0.0000 & 0.004 & 0.0117 \\
ac1 & 0.0000 & 0.0000 & 0.0000 & 0.0000 & 0.0000 & 0.0000 & 0.0000 & 0.0640 & $\mathbf{0 . 8 7 0 0}$ & 0.1670 & 0.0007 & 0.0655 \\
\hline ac2 & 0.0000 & 0.0000 & 0.0000 & 0.0000 & 0.0000 & 0.0000 & 0.0000 & 0.0000 & 0.0650 & $\mathbf{0 . 7 5 0 0}$ & $9 \mathrm{E}-05$ & 0.1827 \\
\hline $\mathrm{N}$ & 21 & 10 & 10 & 68 & 57 & 14 & 17 & 47 & 46 & 12 & &
\end{tabular}

\begin{tabular}{|c|c|c|c|c|c|c|c|c|c|c|c|c|}
\hline \multicolumn{3}{|c|}{ San Benito 2005-2006 } & \multicolumn{10}{|c|}{$\lambda=0.9632 \pm 0.071$} \\
\hline & $\mathbf{P}$ & I & $J$ & Al & All & AIII & ic & jc & ac1 & ac2 & w & $\mathrm{v}$ \\
\hline $\mathbf{P}$ & 0.0000 & 0.0000 & 0.0000 & 2.026 & 7.6800 & 11.000 & 0.0000 & 0.0000 & 1.2320 & 7.4580 & 0.6582 & 0.0000 \\
\hline I & 0.0160 & 0.5000 & 0.0000 & 0.0000 & 0.0000 & 0.0000 & 0.0000 & 0.0000 & 0.0000 & 0.0000 & 0.0226 & $2 \mathrm{E}-05$ \\
\hline J & 0.0000 & 0.0100 & 0.5710 & 0.0000 & 0.0000 & 0.0000 & 0.0000 & 0.0000 & 0.0000 & 0.0000 & 0.0006 & 0.001 \\
\hline Al & 0.0000 & 0.0000 & 0.1430 & 0.8660 & 0.1520 & 0.0000 & 0.0000 & 0.0000 & 0.0000 & 0.0000 & 0.0009 & 0.0029 \\
\hline All & 0.0000 & 0.0000 & 0.0000 & 0.0190 & 0.7420 & 0.3330 & 0.0000 & 0.0000 & 0.0000 & 0.0000 & $9 \mathrm{E}-05$ & 0.0151 \\
\hline AIII & 0.0000 & 0.0000 & 0.0000 & 0.0000 & 0.0150 & 0.6670 & 0.0000 & 0.0000 & 0.0000 & 0.0000 & 0.0000 & 0.0168 \\
\hline ic & 0.0000 & 0.0000 & 0.0000 & 0.0000 & 0.1060 & 0.0000 & 0.0710 & 0.0000 & 0.0000 & 0.0000 & $1 \mathrm{E}-05$ & 0.0256 \\
\hline jc & 0.0000 & 0.0000 & 0.0000 & 0.0000 & 0.0000 & 0.0000 & 0.2140 & 0.6420 & 0.0220 & 0.0000 & 0.0175 & 0.1071 \\
\hline ac1 & 0.0000 & 0.0000 & 0.0000 & 0.0000 & 0.0000 & 0.0000 & 0.0000 & 0.0940 & 0.9330 & 0.1670 & 0.2578 & 0.3699 \\
\hline ac2 & 0.0000 & 0.0000 & 0.0000 & 0.0000 & 0.0000 & 0.0000 & 0.0000 & 0.0000 & 0.0220 & 0.8330 & 0.0424 & 0.4616 \\
\hline $\mathrm{N}$ & 62 & 10 & 7 & 52 & 66 & 9 & 14 & 53 & 45 & 12 & & \\
\hline \multicolumn{3}{|c|}{ San Benito 2006-2007 } & \multicolumn{10}{|c|}{$\lambda=1.0119 \pm 0.252$} \\
\hline & $\mathbf{P}$ & I & $J$ & Al & All & AllI & ic & jc & ac1 & ac2 & w & $\mathbf{v}$ \\
\hline $\mathbf{P}$ & 0.0000 & 0.0000 & 0.0000 & 3.266 & 16.926 & 24.557 & 0.0000 & 0.0000 & 1.9650 & 14.127 & 0.9446 & 0.0002 \\
\hline I & 0.0010 & 0.5000 & 0.0000 & 0.0000 & 0.0000 & 0.0000 & 0.0000 & 0.0000 & 0.0000 & 0.0000 & 0.0018 & 0.1555 \\
\hline$J$ & 0.0000 & 0.5000 & 0.5000 & 0.0000 & 0.0000 & 0.0000 & 0.0000 & 0.0000 & 0.0000 & 0.0000 & 0.0018 & 0.1601 \\
\hline Al & 0.0000 & 0.0000 & 0.5000 & 0.5360 & 0.0000 & 0.0000 & 0.0000 & 0.0000 & 0.0000 & 0.0000 & 0.0019 & 0.1613 \\
\hline All & 0.0000 & 0.0000 & 0.0000 & 0.3570 & 0.7690 & 0.0000 & 0.0000 & 0.0000 & 0.0000 & 0.0000 & 0.0027 & 0.2113 \\
\hline AIII & 0.0000 & 0.0000 & 0.0000 & 0.0000 & 0.1920 & 1.0000 & 0.0000 & 0.0000 & 0.0000 & 0.0000 & 0.0346 & 0.2514 \\
\hline ic & 0.0000 & 0.0000 & 0.0000 & 0.2500 & 0.1790 & 0.1540 & 0.5330 & 0.0230 & 0.0000 & 0.0000 & 0.0026 & 0.0073 \\
\hline jc & 0.0000 & 0.0000 & 0.0000 & 0.0000 & 0.0000 & 0.0000 & 0.4000 & 0.6740 & 0.0410 & 0.0000 & 0.0035 & 0.0088 \\
\hline ac1 & 0.0000 & 0.0000 & 0.0000 & 0.0000 & 0.0000 & 0.0000 & 0.0000 & 0.1630 & 0.7140 & 0.0910 & 0.0030 & 0.0129 \\
\hline ac2 & 0.0000 & 0.0000 & 0.0000 & 0.0000 & 0.0000 & 0.0000 & 0.0000 & 0.0230 & 0.1020 & 0.9090 & 0.0036 & 0.0315 \\
\hline $\mathrm{N}$ & 525 & 2 & 4 & 56 & 52 & 7 & 15 & 43 & 49 & 11 & & \\
\hline
\end{tabular}


Apéndice 1. Continuación

\begin{tabular}{|c|c|c|c|c|c|c|c|c|c|c|c|c|}
\hline \multicolumn{3}{|c|}{ San Bruno 2004-2005 } & \multicolumn{10}{|c|}{$\lambda=0.9848 \pm 0.209$} \\
\hline & $\mathbf{P}$ & I & $J$ & Al & All & AlII & ic & jc & ac1 & ac2 & $w$ & $\mathbf{v}$ \\
\hline $\mathbf{P}$ & 0.0000 & 0.0000 & 0.0000 & 10.440 & 23.828 & 0.0000 & 0.0000 & 0.0000 & 0.8430 & 15.451 & 0.3816 & 0.0000 \\
\hline I & 0.0010 & 0.0010 & 0.0000 & 0.0000 & 0.0000 & 0.0000 & 0.0000 & 0.0000 & 0.0000 & 0.0000 & 0.0186 & 0.0000 \\
\hline J & 0.0000 & 0.0010 & 0.0010 & 0.0000 & 0.0000 & 0.0000 & 0.0000 & 0.0000 & 0.0000 & 0.0000 & $2 \mathrm{E}-05$ & 0.0004 \\
\hline Al & 0.0000 & 0.0000 & 0.0010 & 0.6000 & 0.0000 & 0.0000 & 0.0000 & 0.0000 & 0.0000 & 0.0000 & 0.0000 & 0.4330 \\
\hline All & 0.0000 & 0.0000 & 0.0000 & 0.4000 & 0.8820 & 0.0000 & 0.0000 & 0.0000 & 0.0000 & 0.0000 & 0.0000 & 0.3227 \\
\hline AlII & 0.0000 & 0.0000 & 0.0000 & 0.0000 & 0.1180 & 0.0010 & 0.0000 & 0.0000 & 0.0000 & 0.0000 & 0.0000 & 0.0000 \\
\hline ic & 0.0000 & 0.0000 & 0.0000 & 0.6000 & 0.5290 & 0.0000 & 0.3850 & 0.0000 & 0.0000 & 0.0000 & 0.0000 & 0.0634 \\
\hline jc & 0.0000 & 0.0000 & 0.0000 & 0.0000 & 0.0000 & 0.0000 & 0.6150 & 0.7620 & 0.0000 & 0.0000 & 0.1535 & 0.0619 \\
\hline ac1 & 0.0000 & 0.0000 & 0.0000 & 0.0000 & 0.0000 & 0.0000 & 0.0000 & 0.2380 & 0.9040 & 0.4000 & 0.4463 & 0.0582 \\
\hline ac2 & 0.0000 & 0.0000 & 0.0000 & 0.0000 & 0.0000 & 0.0000 & 0.0000 & 0.0000 & 0.0770 & 0.6000 & 0.0000 & 0.0604 \\
\hline $\mathrm{N}$ & 62 & 10 & 10 & 5 & 17 & 10 & 13 & 21 & 52 & 5 & & \\
\hline
\end{tabular}

\begin{tabular}{|c|c|c|c|c|c|c|c|c|c|c|c|c|}
\hline \multicolumn{3}{|c|}{ San Bruno 2005-2006 } & \multicolumn{10}{|c|}{$\lambda=1.0139 \pm 0.070$} \\
\hline & $\mathbf{P}$ & I & $J$ & $\mathrm{Al}$ & All & AlII & ic & jc & ac1 & ac2 & w & $\mathbf{v}$ \\
\hline $\mathbf{P}$ & 0.0000 & 0.0000 & 0.0000 & 6.6000 & 9.0380 & 1.1880 & 0.0000 & 0.0000 & 0.3080 & 9.7680 & 0.8925 & 0.0000 \\
\hline I & 0.0160 & 0.0010 & 0.0000 & 0.0000 & 0.0000 & 0.0000 & 0.0000 & 0.0000 & 0.0000 & 0.0000 & 0.0143 & 0.0000 \\
\hline J & 0.0000 & 0.0010 & 0.0010 & 0.0000 & 0.0000 & 0.0000 & 0.0000 & 0.0000 & 0.0000 & 0.0000 & $1 \mathrm{E}-05$ & 0.0010 \\
\hline Al & 0.0000 & 0.0000 & 0.0010 & 1.0000 & 0.0000 & 0.0000 & 0.0000 & 0.0000 & 0.0000 & 0.0000 & $3 \mathrm{E}-05$ & 0.9961 \\
\hline All & 0.0000 & 0.0000 & 0.0000 & 0.0000 & 0.9410 & 0.0000 & 0.0000 & 0.0000 & 0.0000 & 0.0000 & 0.0000 & 0.0018 \\
\hline AIII & 0.0000 & 0.0000 & 0.0000 & 0.0000 & 0.0590 & 1.0000 & 0.0000 & 0.0000 & 0.0000 & 0.0000 & 0.0000 & $5 \mathrm{E}-05$ \\
\hline ic & 0.0000 & 0.0000 & 0.0000 & 1.6670 & 0.4120 & 0.0000 & 0.6670 & 0.0000 & 0.0000 & 0.0000 & 0.0002 & 0.0003 \\
\hline jc & 0.0000 & 0.0000 & 0.0000 & 0.0000 & 0.0000 & 0.0000 & 0.3330 & 0.9000 & 0.0000 & 0.0000 & 0.0006 & 0.0003 \\
\hline ac1 & 0.0000 & 0.0000 & 0.0000 & 0.0000 & 0.0000 & 0.0000 & 0.0000 & 0.1000 & 0.9440 & 0.0000 & 0.0010 & 0.0003 \\
\hline ac2 & 0.0000 & 0.0000 & 0.0000 & 0.0000 & 0.0000 & 0.0000 & 0.0000 & 0.0000 & 0.0370 & 1.0000 & 0.0914 & 0.0004 \\
\hline $\mathrm{N}$ & 6 & 10 & 10 & 3 & 17 & 2 & 15 & 30 & 54 & 3 & & \\
\hline
\end{tabular}

\begin{tabular}{|c|c|c|c|c|c|c|c|c|c|c|c|c|}
\hline \multicolumn{3}{|c|}{ San Bruno 2006-2007 } & \multicolumn{10}{|c|}{$\lambda=0.6255 \pm 0.210$} \\
\hline & $\mathbf{P}$ & I & J & $\mathrm{Al}$ & AlI & AlII & ic & jc & ac1 & ac2 & $\mathbf{w}$ & $\mathbf{v}$ \\
\hline $\mathbf{P}$ & 0.0000 & 0.0000 & 0.0000 & 8.5950 & 14.822 & 0.0000 & 0.0000 & 0.0000 & 0.5350 & 16.961 & 0.9607 & 0.0000 \\
\hline I & 0.0010 & 0.0100 & 0.0000 & 0.0000 & 0.0000 & 0.0000 & 0.0000 & 0.0000 & 0.0000 & 0.0000 & 0.0015 & 0.0000 \\
\hline$J$ & 0.0000 & 0.0100 & 0.0100 & 0.0000 & 0.0000 & 0.0000 & 0.0000 & 0.0000 & 0.0000 & 0.0000 & $2 \mathrm{E}-05$ & $6 \mathrm{E}-05$ \\
\hline Al & 0.0000 & 0.0000 & 0.0100 & 0.1250 & 0.1110 & 0.0000 & 0.0000 & 0.0000 & 0.0000 & 0.0000 & 0.0000 & 0.0039 \\
\hline All & 0.0000 & 0.0000 & 0.0000 & 0.5000 & 0.5000 & 0.0000 & 0.0000 & 0.0000 & 0.0000 & 0.0000 & 0.0000 & 0.0037 \\
\hline AIII & 0.0000 & 0.0000 & 0.0000 & 0.0000 & 0.0560 & 0.0010 & 0.0000 & 0.0000 & 0.0000 & 0.0000 & 0.0000 & 0.0000 \\
\hline ic & 0.0000 & 0.0000 & 0.0000 & 0.5000 & 0.3890 & 0.0000 & 0.0590 & 0.0000 & 0.0370 & 0.0000 & 0.0000 & 0.0005 \\
\hline jc & 0.0000 & 0.0000 & 0.0000 & 0.0000 & 0.0000 & 0.0000 & 0.0590 & 0.0280 & 0.0370 & 0.0000 & 0.0000 & 0.0051 \\
\hline ac1 & 0.0000 & 0.0000 & 0.0000 & 0.0000 & 0.0000 & 0.0000 & 0.0000 & 0.1110 & 0.0370 & 0.0000 & 0.0000 & 0.0292 \\
\hline ac2 & 0.0000 & 0.0000 & 0.0000 & 0.0000 & 0.0000 & 0.0000 & 0.0000 & 0.0000 & 0.0190 & 0.667 & 0.0378 & 0.9575 \\
\hline $\mathrm{N}$ & 525 & 10 & 10 & 8 & 18 & 10 & 17 & 36 & 54 & 3 & & \\
\hline
\end{tabular}


Merari Ferrer, Rafael Durán, Martha Méndez, Alfredo Dorantes y Gabriel Dzib.

Apéndice 1. Continuación

\begin{tabular}{|c|c|c|c|c|c|c|c|c|c|c|c|c|}
\hline \multicolumn{3}{|c|}{ San Felipe 2004-2005 } & \multicolumn{10}{|c|}{$\lambda=0.9964 \pm 0.019$} \\
\hline & $\mathbf{P}$ & I & $J$ & $\mathrm{Al}$ & All & AIII & ic & jc & ac1 & ac2 & w & $\mathbf{v}$ \\
\hline $\mathbf{P}$ & 0.0000 & 0.0000 & 0.0000 & 0.2610 & 2.4860 & 15.776 & 0.0000 & 0.0000 & 0.7690 & 5.3360 & 0.8088 & 0.0000 \\
\hline I & 0.0010 & 0.0100 & 0.0000 & 0.0000 & 0.0000 & 0.0000 & 0.0000 & 0.0000 & 0.0000 & 0.0000 & 0.0392 & 0.00001 \\
\hline J & 0.0000 & 0.0100 & 0.0100 & 0.0000 & 0.0000 & 0.0000 & 0.0000 & 0.0000 & 0.0000 & 0.0000 & 0.0004 & 0.0006 \\
\hline Al & 0.0000 & 0.0000 & 0.0100 & 0.6250 & 0.0000 & 0.0000 & 0.0000 & 0.0000 & 0.0000 & 0.0000 & 0.00001 & 0.0610 \\
\hline All & 0.0000 & 0.0000 & 0.0000 & 0.1250 & 0.9520 & 0.0000 & 0.0000 & 0.0000 & 0.0000 & 0.0000 & 0.00003 & 0.1624 \\
\hline AIII & 0.0000 & 0.0000 & 0.0000 & 0.0000 & 0.0480 & 0.8890 & 0.0000 & 0.0000 & 0.0000 & 0.0000 & 0.00001 & 0.0408 \\
\hline ic & 0.0000 & 0.0000 & 0.0000 & 0.0630 & 0.1430 & 0.1110 & 0.6000 & 0.0000 & 0.0000 & 0.0000 & 0.00001 & 0.0408 \\
\hline jc & 0.0000 & 0.0000 & 0.0000 & 0.0000 & 0.0000 & 0.0000 & 0.1000 & 0.4000 & 0.0530 & 0.0000 & 0.0000 & 0.1632 \\
\hline ac1 & 0.0000 & 0.0000 & 0.0000 & 0.0000 & 0.0000 & 0.0000 & 0.0000 & 0.4000 & 0.8420 & 0.0000 & 0.0000 & 0.2448 \\
\hline ac2 & 0.0000 & 0.0000 & 0.0000 & 0.0000 & 0.0000 & 0.0000 & 0.0000 & 0.0000 & 0.1050 & 1.0000 & 0.1515 & 0.2861 \\
\hline $\mathrm{N}$ & 62 & 10 & 10 & 16 & 21 & 9 & 10 & 10 & 19 & 9 & & \\
\hline
\end{tabular}

\begin{tabular}{llllllllllllll}
\hline \multicolumn{1}{c}{ San Felipe 2005-2006 } & \multicolumn{1}{l}{$\lambda=\mathbf{0 . 9 9 8 6} \pm \mathbf{0 . 0 0 1}$} \\
\hline & $\mathbf{P}$ & $\mathbf{I}$ & $\mathbf{J}$ & $\mathbf{A l}$ & All & Alll & ic & jc & ac1 & ac2 & $\mathbf{w}$ & $\mathbf{v}$ \\
$\mathbf{P}$ & $\mathbf{0 . 0 0 0 0}$ & 0.0000 & 0.0000 & 0.1580 & 0.9000 & 5.9840 & 0.0000 & 0.0000 & 0.2770 & 1.6560 & 0.8449 & 0.0000 \\
$\mathbf{I}$ & 0.0160 & $\mathbf{0 . 0 1 0 0}$ & 0.0000 & 0.0000 & 0.0000 & 0.0000 & 0.0000 & 0.0000 & 0.0000 & 0.0000 & 0.0136 & 0.0001 \\
J & 0.0000 & 0.0100 & $\mathbf{0 . 0 1 0 0}$ & 0.0000 & 0.0000 & 0.0000 & 0.0000 & 0.0000 & 0.0000 & 0.0000 & 0.00014 & 0.0011 \\
AI & 0.0000 & 0.0000 & 0.0100 & $\mathbf{0 . 8 0 0 0}$ & 0.0000 & 0.0000 & 0.0000 & 0.0000 & 0.0000 & 0.0000 & 0.0001 & 0.1109 \\
All & 0.0000 & 0.0000 & 0.0000 & 0.1000 & $\mathbf{0 . 7 2 7 0}$ & 0.0000 & 0.0000 & 0.0000 & 0.0000 & 0.0000 & 0.0000 & 0.2219 \\
AIII & 0.0000 & 0.0000 & 0.0000 & 0.0000 & 0.0910 & $\mathbf{1 . 0 0 0 0}$ & 0.0000 & 0.0000 & 0.0000 & 0.0000 & 0.1412 & 0.6659 \\
ic & 0.0000 & 0.0000 & 0.0000 & $\mathbf{0 . 1 0 0 0}$ & $\mathbf{0 . 0 9 1 0}$ & $\mathbf{0 . 0 0 0 0}$ & $\mathbf{0 . 6 0 0 0}$ & 0.0000 & 0.0000 & 0.0000 & 0.0000 & 0.0000 \\
jc & 0.0000 & 0.0000 & 0.0000 & 0.0000 & 0.0000 & 0.0000 & 0.0840 & $\mathbf{0 . 1 6 7 0}$ & 0.0000 & 0.0000 & 0.0000 & 0.0000 \\
ac1 & 0.0000 & 0.0000 & 0.0000 & 0.0000 & 0.0000 & 0.0000 & 0.0000 & 0.1670 & $\mathbf{0 . 3 5 0 0}$ & 0.0910 & 0.0000 & 0.0000 \\
\hline ac2 & 0.0000 & 0.0000 & 0.0000 & 0.0000 & 0.0000 & 0.0000 & 0.0000 & 0.0000 & 0.0500 & $\mathbf{0 . 8 1 8 0}$ & 0.0000 & 0.0000 \\
\hline $\mathrm{N}$ & 6 & 10 & 10 & 10 & 22 & 9 & 12 & 6 & 20 & 11 & &
\end{tabular}

\begin{tabular}{|c|c|c|c|c|c|c|c|c|c|c|c|c|}
\hline \multicolumn{3}{|c|}{ San Felipe 2006-2007 } & \multicolumn{10}{|c|}{$\lambda=1.0031 \pm 0.014$} \\
\hline & $\mathbf{P}$ & $I$ & $J$ & Al & All & AIII & ic & jc & ac1 & ac2 & w & $\mathbf{v}$ \\
\hline $\mathbf{P}$ & 0.0000 & 0.0000 & 0.0000 & 0.3440 & 2.0220 & 8.5010 & 0.0000 & 0.0000 & 0.9630 & 3.5140 & 0.68426 & 0.00000 \\
\hline I & 0.0010 & 0.0100 & 0.0000 & 0.0000 & 0.0000 & 0.0000 & 0.0000 & 0.0000 & 0.0000 & 0.0000 & 0.00069 & 0.00001 \\
\hline J & 0.0000 & 0.0100 & 0.0100 & 0.00000 & 0.0000 & 0.0000 & 0.0000 & 0.0000 & 0.0000 & 0.0000 & 0.00001 & 0.00097 \\
\hline Al & 0.0000 & 0.0000 & 0.0100 & 0.5000 & 0.0000 & 0.0000 & 0.0000 & 0.0000 & 0.0000 & 0.0000 & 0.00000 & 0.09640 \\
\hline All & 0.0000 & 0.0000 & 0.0000 & 0.2500 & 0.8240 & 0.2703 & 0.0000 & 0.0000 & 0.0000 & 0.0000 & 0.00000 & 0.19279 \\
\hline AllI & 0.0000 & 0.0000 & 0.0000 & 0.0000 & 0.0580 & 0.7207 & 0.0000 & 0.0000 & 0.0000 & 0.0000 & 0.00000 & 0.19279 \\
\hline ic & 0.0000 & 0.0000 & 0.0000 & 0.0000 & 0.1760 & 0.0000 & 0.9170 & 0.0000 & 0.0000 & 0.0000 & 0.00000 & 0.12926 \\
\hline jc & 0.0000 & 0.0000 & 0.0000 & 0.0000 & 0.0000 & 0.0000 & 0.0830 & 0.7500 & 0.0000 & 0.0000 & 0.00000 & 0.12926 \\
\hline ac1 & 0.0000 & 0.0000 & 0.0000 & 0.0000 & 0.0000 & 0.0000 & 0.0000 & 0.2500 & 0.9000 & 0.1110 & 0.16573 & 0.12926 \\
\hline ac2 & 0.0000 & 0.0000 & 0.0000 & 0.0000 & 0.0000 & 0.0000 & 0.0000 & 0.0000 & 0.1000 & 0.8890 & 0.14931 & 0.12926 \\
\hline $\mathrm{N}$ & 525 & 10 & 10 & 0 & 17 & 11 & 12 & 4 & 10 & $J$ & & \\
\hline
\end{tabular}


Apéndice 1. Continuación

\begin{tabular}{|c|c|c|c|c|c|c|c|c|c|c|c|c|}
\hline \multicolumn{3}{|c|}{ Las Coloradas 2004-2005 } & \multicolumn{10}{|c|}{$\lambda=0.9876 \pm 0.021$} \\
\hline & $\mathbf{P}$ & I & $J$ & Al & All & AIII & ic & jc & ac1 & ac2 & w & v \\
\hline $\mathbf{P}$ & 0.0000 & 0.0000 & 0.0000 & 54.280 & 24.590 & 61.691 & 0.0000 & 0.0000 & 6.7280 & 36.680 & 0.9010 & 0.0000 \\
\hline I & 0.0010 & 0.0010 & 0.0000 & 0.0000 & 0.0000 & 0.0000 & 0.0000 & 0.0000 & 0.0000 & 0.0000 & 0.0450 & 0.0000 \\
\hline$J$ & 0.0000 & 0.000 & 0.0010 & 0.0000 & 0.0000 & 0.0000 & 0.0000 & 0.0000 & 0.0000 & 0.0000 & $5 \mathrm{E}-05$ & $5 \mathrm{E}-04$ \\
\hline Al & 0.0000 & 0.0000 & 0.0010 & 0.9000 & 0.2220 & 0.0000 & 0.0000 & 0.0000 & 0.0000 & 0.0000 & 0.0020 & 0.4410 \\
\hline All & 0.0000 & 0.0000 & 0.0000 & 0.1000 & 0.4440 & 0.2730 & 0.0000 & 0.0000 & 0.0000 & 0.0000 & $6 \mathrm{E}-04$ & 0.2560 \\
\hline AlII & 0.0000 & 0.0000 & 0.0000 & 0.0000 & 0.1110 & 0.7270 & 0.0000 & 0.0000 & 0.0000 & 0.0000 & $3 \mathrm{E}-04$ & 0.3020 \\
\hline ic & 0.0000 & 0.0000 & 0.0000 & 0.0000 & 3.0000 & 0.7270 & 0.7270 & 0.0000 & 0.0000 & 0.0000 & 0.0080 & $1 \mathrm{E}-05$ \\
\hline jc & 0.0000 & 0.0000 & 0.0000 & 0.0000 & 0.0000 & 0.0000 & 0.2270 & 0.6360 & 0.0560 & 0.0000 & 0.0090 & $1 \mathrm{E}-05$ \\
\hline ac1 & 0.0000 & 0.0000 & 0.0000 & 0.0000 & 0.0000 & 0.0000 & 0.0000 & 0.3180 & 0.6670 & 0.1430 & 0.0180 & $1 \mathrm{E}-05$ \\
\hline ac2 & 0.0000 & 0.0000 & 0.0000 & 0.0000 & 0.0000 & 0.0000 & 0.0000 & 0.0000 & 0.2220 & 0.7140 & 0.0160 & $1 \mathrm{E}-05$ \\
\hline $\mathrm{N}$ & 62 & 10 & 10 & 10 & 9 & 11 & 22 & 22 & 18 & 14 & & \\
\hline \multicolumn{3}{|c|}{ Las Coloradas 2005-2006 } & \multicolumn{10}{|c|}{$\lambda=0.9369 \pm 0.042$} \\
\hline & $\mathbf{P}$ & I & $J$ & Al & AlI & AIII & ic & jc & ac1 & ac2 & w & $\mathbf{v}$ \\
\hline $\mathbf{P}$ & 0.0000 & 0.0000 & 0.0000 & 6.8640 & 11.993 & 28.600 & 0.0000 & 0.0000 & 2.1870 & 13.917 & 0.8870 & 0.0000 \\
\hline I & 0.0160 & 0.0010 & 0.0000 & 0.0000 & 0.0000 & 0.0000 & 0.0000 & 0.0000 & 0.0000 & 0.0000 & 0.0150 & 0.0000 \\
\hline$J$ & 0.0000 & 0.0010 & 0.0010 & 0.0000 & 0.0000 & 0.0000 & 0.0000 & 0.0000 & 0.0000 & 0.0000 & $2 \mathrm{E}-05$ & $1 \mathrm{E}-04$ \\
\hline Al & 0.0000 & 0.0000 & 0.0010 & 0.3330 & 0.2860 & 0.0000 & 0.0000 & 0.0000 & 0.0000 & 0.0000 & 0.0000 & 0.1170 \\
\hline All & 0.0000 & 0.0000 & 0.0000 & 0.3330 & 0.1430 & 0.2220 & 0.0000 & 0.0000 & 0.0000 & 0.0000 & 0.0000 & 0.2080 \\
\hline AlII & 0.0000 & 0.0000 & 0.0000 & 0.0000 & 0.5710 & 0.6670 & 0.0000 & 0.0000 & 0.0000 & 0.0000 & 0.0000 & 0.1800 \\
\hline ic & 0.0000 & 0.0000 & 0.0000 & 0.0000 & 0.7140 & 0.0000 & 0.0950 & 0.0000 & 0.0000 & 0.0000 & 0.0000 & 0.0370 \\
\hline jc & 0.0000 & 0.0000 & 0.0000 & 0.0000 & 0.0000 & 0.0000 & 0.1900 & 0.6500 & 0.0000 & 0.0000 & 0.0000 & 0.1610 \\
\hline ac1 & 0.0000 & 0.0000 & 0.0000 & 0.0000 & 0.0000 & 0.0000 & 0.0000 & 0.2500 & 0.7620 & 0.1430 & 0.0460 & 0.1770 \\
\hline ac2 & 0.0000 & 0.0000 & 0.0000 & 0.0000 & 0.0000 & 0.0000 & 0.0000 & 0.0000 & 0.2380 & 0.7140 & 0.0520 & 0.1200 \\
\hline $\mathrm{N}$ & 6 & 10 & 10 & 3 & 7 & 9 & 21 & 20 & 21 & 14 & & \\
\hline \multicolumn{3}{|c|}{ Las Coloradas 2006-2007 } & \multicolumn{10}{|c|}{$\lambda=1.0313 \pm 0.042$} \\
\hline & $\mathbf{P}$ & I & $J$ & Al & All & AlII & ic & jc & ac1 & ac2 & w & $\mathbf{v}$ \\
\hline $\mathbf{P}$ & 0.0000 & 0.0000 & 0.0000 & 11.918 & 36.440 & 44.690 & 0.0000 & 0.0000 & 3.3230 & 22.550 & 0.9190 & 0.0000 \\
\hline I & 0.0010 & 0.0010 & 0.0000 & 0.0000 & 0.0000 & 0.0000 & 0.0000 & 0.0000 & 0.0000 & 0.0000 & $9 \mathrm{E}-04$ & 0.0000 \\
\hline$J$ & 0.0000 & 0.0010 & 0.0010 & 0.0000 & 0.0000 & 0.0000 & 0.0000 & 0.0000 & 0.0000 & 0.0000 & 0.0000 & $3 \mathrm{E}-04$ \\
\hline Al & 0.0000 & 0.0000 & 0.0010 & 0.1000 & 0.0000 & 0.0000 & 0.0000 & 0.0000 & 0.0000 & 0.0000 & 0.0000 & 0.2500 \\
\hline All & 0.0000 & 0.0000 & 0.0000 & 0.6000 & 0.0100 & 0.1000 & 0.0000 & 0.0000 & 0.0000 & 0.0000 & $5 \mathrm{E}-04$ & 0.3750 \\
\hline AlII & 0.0000 & 0.0000 & 0.0000 & 0.0000 & 0.9900 & 0.9000 & 0.0000 & 0.0000 & 0.0000 & 0.0000 & 0.0050 & 0.3750 \\
\hline ic & 0.0000 & 0.0000 & 0.0000 & 0.0000 & 4.5000 & 1.3000 & 0.0520 & 0.0000 & 0.0000 & 0.0000 & 0.0100 & 0.0000 \\
\hline jc & 0.0000 & 0.0000 & 0.0000 & 0.0000 & 0.0000 & 0.0000 & 0.6320 & 0.3330 & 0.0000 & 0.0000 & 0.0090 & 0.0000 \\
\hline ac1 & 0.0000 & 0.0000 & 0.0000 & 0.0000 & 0.0000 & 0.0000 & 0.1580 & 0.3330 & 0.7920 & 0.0670 & 0.0300 & 0.0000 \\
\hline ac2 & 0.0000 & 0.0000 & 0.0000 & 0.0000 & 0.0000 & 0.0000 & 0.0000 & 0.0000 & 0.1670 & 0.8000 & 0.0250 & 0.0000 \\
\hline $\mathrm{N}$ & 525 & 10 & 10 & 10 & 9 & 11 & 22 & 22 & 18 & 14 & & \\
\hline
\end{tabular}

